\title{
SUPRACONVERGENCE AND SUPERCLOSENESS OF A DISCRETISATION FOR ELLIPTIC THIRD-KIND BOUNDARY-VALUE PROBLEMS ON POLYGONAL DOMAINS
}

\author{
E. EMMRICH ${ }^{1}$
}

\begin{abstract}
The third-kind boundary-value problem for a second-order elliptic equation on a polygonal domain with variable coefficients, mixed derivatives, and first-order terms is approximated by a linear finite element method with first-order accurate quadrature. The corresponding bilinear form does not need to be strongly positive. The discretisation is equivalent to a finite difference scheme.

Although the discretisation is in general only first-order consistent, supraconvergence, i.e., convergence of higher order, is shown to take place even on nonuniform grids. Local error estimates of optimal order $\min (s, 3 / 2)$ (with a logarithmic factor if $s=3 / 2$ ) in the $H^{1}(\Omega)$-norm are proved for $s \in(1 / 2,2]$ if the exact solution is in the Sobolev Slobodetskij space $H^{1+s}(\Omega)$. If neither oblique boundary sections nor mixed derivatives occur, then the optimal order $s$ is achieved. The supraconvergence result is equivalent to the supercloseness of the gradient.
\end{abstract}

2000 Mathematics Subject Classification: 65N12, 65N15, 65N06, 65N30.

Keywords: elliptic PDE, fully discrete FEM, FDM, nonuniform grid, supraconvergence, supercloseness of gradient.

\section{Introduction}

We consider the discretisation of the differential equation

$$
A u:=-\left(a u_{x}\right)_{x}-\left(b u_{x}\right)_{y}-\left(b u_{y}\right)_{x}-\left(c u_{y}\right)_{y}+d u_{x}+e u_{y}+f u=g \quad \text { in } \Omega \subset \mathbb{R}^{2}
$$

with variable coefficients subject to the Robin boundary conditions

$$
B u:=a u_{x} \eta_{x}+b u_{x} \eta_{y}+b u_{y} \eta_{x}+c u_{y} \eta_{y}+\alpha u=\psi \quad \text { on } \Gamma:=\partial \Omega
$$

where $\Omega$ is a simple polygonal domain and $\left(\eta_{x}, \eta_{y}\right)$ denotes the outer normal on $\Gamma$. The coefficients and the right-hand sides are assumed to be sufficiently smooth. Moreover, $A$ is assumed to be uniformly elliptic and the corresponding homogeneous problem with $g=\psi=0$ is supposed to possess the trivial solution $u=0$, only. It is, however, not necessary to assume strong positiveness of the corresponding bilinear form.

The discretisation is obtained from linear finite elements on a triangulation $\mathcal{T}_{H}$ of $\Omega$, which relies upon a nonuniform rectangular grid $\bar{\Omega}_{H}$, in combination with an appropriate

\footnotetext{
${ }^{1}$ Technische Universität Berlin, Institut für Mathematik, Straße des 17. Juni 136, 10623 Berlin, Germany. E-mail: emmrich@math.tu-berlin.de
} 
first-order quadrature. This fully discrete finite element method is equivalent to a finite difference method on $\bar{\Omega}_{H}$.

Although the scheme is in general only first-order consistent, we prove higher-order convergence. Let $u_{H}$ denote the finite difference solution, $P_{H}$ - the piecewise linear interpolation with respect to $\mathcal{T}_{H}$ (note that $P_{H} u_{H}$ then is the corresponding finite element solution), $R_{H}$ - the pointwise restriction on $\bar{\Omega}_{H}$, and $\|\cdot\|_{1,2}$ - the usual $H^{1}(\Omega)$-norm. For a quasiuniform sequence of grids $\bar{\Omega}_{H}$ with a maximum meshsize $H_{\max }$ tending to zero, we derive a local error estimate from which the global estimate $\left\|P_{H} R_{H} u-P_{H} u_{H}\right\|_{1,2}=\mathcal{O}\left(H_{\max }^{\min (s, 3 / 2)}\right)$ for $s \in(1 / 2,2], s \neq 3 / 2$, and $\left\|P_{H} R_{H} u-P_{H} u_{H}\right\|_{1,2}=\mathcal{O}\left(H_{\max }^{3 / 2} \log H_{\max }^{-1}\right)$ for $s=3 / 2$, respectively, follows if $u \in H^{1+s}(\Omega)$. For $s \in\{1,2\}$, the estimate even holds without the assumption of quasi-uniformity. If $\Omega$ is a union of rectangles then for $s=3 / 2$, the logarithmic factor does not appear. If, moreover, there are no mixed derivatives, then even the optimal order $s$ can be shown. As a consequence, a global estimate of order $\mathcal{O}\left(H_{\max }^{s}\right)$ is obtained for $s \in[1,2]$ even for an arbitrary sequence of nonuniform grids if there are no oblique boundary sections and no mixed derivatives. Again by interpolation, a global estimate of order $\mathcal{O}\left(H_{\max }^{(1+s) / 2}\right)$ follows for an arbitrary sequence of nonuniform grids in case $\Omega$ is a union of rectangles. By assuming $u \in H^{1+s}(\Omega) \cap W^{2, \infty}(\Omega)$, local error estimates can be proved that lead to global estimates of order $\mathcal{O}\left(H_{\max }^{\min (s, 3 / 2)}\right)$ without the logarithmic factor even if oblique boundary sections and mixed derivatives occur. Note that the supraconvergence results also show the supercloseness property for the gradient in the context of finite elements.

The super- and supraconvergence of finite elements and finite difference solutions, respectively, have been considered by many authors; we refer, in particular, to the pioneering work [44] as well as to $[7,22,28,31,50,53]$ and the references cited therein.

Besides elliptic problems, also other types of equations have been considered; without being exhaustive, we refer to $[5,40,48]$ for parabolic equations, to $[4,23,51]$ for second-order hyperbolic equations, to [43] for first-order hyperbolic equations, to [14] for the Korteweg-de Vries equation, and to [39] for other semi- and quasilinear time-dependent problems. Recently, also the supraconvergence of finite volume discretisations for linear advection problems has been studied (see [45]).

Finite difference approximations of linear second-order elliptic equations with Dirichlet boundary conditions have been analysed, mostly by means of function space interpolation techniques and by measuring the error in a discrete Sobolev norm, in [6,21,24-26, 34, 41, 49] (and other papers by the same authors), see also [54] for a finite element discretisation on a nonuniform rectangular partition. In order to obtain higher-order convergence on nonuniform grids, often a correction is added to the standard finite difference scheme on a uniform grid. This is different from the results of $[3,11-13]$ and of this paper where no correction is needed.

The supercloseness property, which follows from the supraconvergence results, plays an important rôle in postprocessing, recovery techniques for the gradient, and a posteriori error estimates (see $[1,2,8,9,16-19,29,30,35,36,38,52]$ and the references cited therein). In all these papers, either a uniform grid or a smooth transformation of a uniform grid (cf., e.g., [35]) has to be assumed in order to obtain optimal second-order convergence for the gradient in the $L^{2}(\Omega)$-norm if $u \in H^{3}(\Omega)$. Moreover, the coefficients are often supposed to be constant, the domain is often the unit square or with a smooth boundary, and the quadrature used in [35] is of second order, which differs from the first-order quadrature studied in this paper. Except for $[17,36]$, only Dirichlet boundary conditions are considered. The order of convergence in [17] is $3 / 2$ for Neumann boundary conditions if (except near the smooth boundary) a uniform grid is used. Also in the tables in [36], one can find order $3 / 2$ but for bilinear elements. In 
the recent paper [1], again mixed derivatives and Neumann boundary conditions are allowed; the maximum order of convergence proven there is $\mathcal{O}\left(h^{2} \log ^{1 / 2} h^{-1}\right)$ if the exact solution is in $W^{3, \infty}(\Omega)$ and if the triangulation is quasi-uniform and most of the adjacent triangles fulfill the so-called $\mathcal{O}\left(h^{2}\right)$-parallelogram property. Recently, the supercloseness property has been studied in $[37,47]$ for the finite element discretisation of the Poisson and convection-diffusion equation with Dirichlet boundary conditions relying on quadrilateral nonconforming elements even on nonuniform grids.

In [27], a particular finite difference discretisation of (1.1), (1.2) with mixed derivatives but without lower-order terms was considered on an equidistant partition of the unit square and global estimates for the error measured in the discrete $H^{1}$-norm of order $\mathcal{O}\left(h^{2} \log ^{3 / 2} h^{-1}\right)$ are obtained if the exact solution is in $H^{3}(\Omega)$. Preceding results can be found in [33, 46], where the finite difference method on a uniform grid in [33] for (1.1), (1.2) without lower-order terms and in [46] for the Poisson equation with third-kind boundary conditions, respectively, has been studied on the unit square. The error measured in the discrete $H^{1}$-norm is shown to be of order $\mathcal{O}\left(h^{\min (s, 3 / 2)}\right)(s \in(1 / 2,2], s \neq 3 / 2)$ and $\mathcal{O}\left(h^{3 / 2} \log h^{-1}\right)(s=3 / 2)$ if $u \in H^{1+s}(\Omega)$.

Our paper is to some extent a continuation of [3,11-13]: In [12], the authors deal with the differential operator (1.1) on a simple polygonal domain subject to Dirichlet boundary conditions instead of (1.2) and show a local error estimate for $\left\|P_{H} R_{H} u-P_{H} u_{H}\right\|_{1,2}$ of second order on arbitrary nonuniform grids if $u \in \mathcal{C}^{4}(\bar{\Omega})$ and if there are no oblique boundary parts in the case $b \neq 0$. In this latter case, the convergence order reduces to $\mathcal{O}\left(H_{\max }^{3 / 2}\right)$ if $\Gamma$ contains oblique parts. In [13], again (1.1) (but with first-order terms in divergence form) is considered on a simple polygonal domain subject to Dirichlet boundary conditions and local error estimates are proved showing first- and second-order convergence if $u \in H^{2}(\Omega)$ and $u \in H^{3}(\Omega)$, respectively, and if there are no oblique boundary parts or if $b=0$. In the case that $\Gamma$ possesses oblique parts and $b \neq 0$, again order $3 / 2$ can be retained if $u \in H^{3}(\Omega) \cap \mathcal{C}^{2}(\bar{\Omega})$ and, by interpolation, order $\mathcal{O}\left(H_{\max }^{(1+s) / 2-\varepsilon}\right)$ with arbitrary $\varepsilon>0$ can be shown for $u \in H^{1+s}(\Omega)(s \in(1,2])$.

For the third-kind boundary-value problem (1.1), (1.2) on a domain $\Omega$ that is the union of rectangles but without mixed or first-order derivatives $(b=d=e=0)$, local error estimates showing supraconvergence of order $s$ for $s \in(1 / 2,2]$ if $u \in H^{1+s}(\Omega)$ are proved in [11]. Preliminary results for the one-dimensional case were obtained in [3]. The aim of this paper is to generalise the results of [11] by admitting also mixed and first-order derivatives in the differential operator $A$ as well as a polygonal domain.

An essential step here and in the analysis in [11-13] is the fact that the discretisation can be regarded as a fully discrete finite element method with quadrature but also as a finite difference method.

\section{Discretisation and error estimates}

The variational problem corresponding to (1.1), (1.2) reads as

$$
\text { find } u \in H^{1}(\Omega) \text { such that } A(u, v)=(g, v)+(\psi, v)_{\Gamma} \text { for all } v \in H^{1}(\Omega)
$$

with the sesquilinear form

$$
\begin{gathered}
A(v, w):=\left(a v_{x}, w_{x}\right)+\left(b v_{x}, w_{y}\right)+\left(b v_{y}, w_{x}\right)+\left(c v_{y}, w_{y}\right)+ \\
+\left(d v_{x}, w\right)+\left(e v_{y}, w\right)+(f v, w)+(\alpha v, w)_{\Gamma}, \quad v, w \in H^{1}(\Omega) .
\end{gathered}
$$


We employ the usual notation for Lebesgue-, Sobolev-, Sobolev — Slobodetskij spaces and spaces of continuously differentiable functions. In particular, we denote by $(\cdot, \cdot)$ and $(\cdot, \cdot)_{\Gamma}$ the inner product on $L^{2}(\Omega)$ and $L^{2}(\Gamma)$, respectively, and by $\|\cdot\|_{r, p, D}$ and $|\cdot|_{r, p, D}$ the usual norm and seminorm on $W^{r, p}(D)$, respectively, for a domain $D$ (where we omit the subscript $D$ if $D=\Omega$ and set $\left.H^{r}(D)=W^{r, 2}(D)\right)$. For properties of the aforementioned function spaces and embedding theorems, we refer in particular to [42] (cf. also [32, pp. 385ff.]). As the boundary $\Gamma$ is only Lipschitz, the norm of Sobolev — Slobodetskij spaces on $\Gamma$ shall be defined through summing up over disjoint straight boundary sections, which is different from the usual definition. In particular, the continuous embedding $H^{r}(\Omega) \hookrightarrow H^{r-1 / 2}(\Gamma)$ then holds true for all $r>1 / 2$.

For the discretisation, let $\boldsymbol{h}=\left\{h_{j}\right\}_{j \in \mathbb{Z}}$ and $\boldsymbol{k}=\left\{k_{\ell}\right\}_{\ell \in \mathbb{Z}}$ be two sequences of positive real numbers and consider the two-dimensional grid $\mathbb{R}_{H}:=\mathbb{R}_{\boldsymbol{h}}^{x} \times \mathbb{R}_{\boldsymbol{k}}^{y}$, where

$$
\mathbb{R}_{h}^{x}:=\left\{x_{j} \in \mathbb{R}: x_{j+1}:=x_{j}+h_{j}, \quad j \in \mathbb{Z}\right\}, \quad \mathbb{R}_{\boldsymbol{k}}^{y}:=\left\{y_{\ell} \in \mathbb{R}: y_{\ell+1}:=y_{\ell}+k_{\ell}, \quad \ell \in \mathbb{Z}\right\}
$$

for $x_{0}, y_{0} \in \mathbb{R}$ given. We define

$$
\Omega_{H}:=\Omega \cap \mathbb{R}_{H}, \quad \Gamma_{H}:=\Gamma \cap \mathbb{R}_{H}, \quad \bar{\Omega}_{H}:=\bar{\Omega} \cap \mathbb{R}_{H}=\Omega_{H} \cup \Gamma_{H} .
$$

Moreover, let $x_{j+1 / 2}:=x_{j}+h_{j} / 2=x_{j+1}-h_{j} / 2:=x_{(j+1)-1 / 2}$ with an analogous notation for the $y$-direction.

Throughout this paper, we impose the following assumptions:

(H1) The coefficients in $A$ and $B$ as well as $\psi$ are sufficiently smooth such that all are at least uniformly continuous and $g \in L^{2}(\Omega)$.

(H2) The differential operator $A$ is uniformly elliptic in $\Omega$ and the corresponding homogeneous variational problem $(2.1)$ is uniquely solvable.

(H3) The vertices of $\Omega$ are in $\Gamma_{H}$. The triangulation $\mathcal{T}_{H}$ is a set of open triangles in which the vertices are the grid points of $\bar{\Omega}_{H}$. The intersection of $\Gamma$ with a rectangle $\square:=\left(x_{j}, x_{j+1}\right) \times\left(y_{\ell}, y_{\ell+1}\right)$ is either empty or a diagonal of $\square$.

(H4) The set $\Lambda$ describes the sequence $\left\{\bar{\Omega}_{H}\right\}_{H \in \Lambda}$ of grids with $H_{\max }:=\max \left\{h_{j}, k_{\ell}: j, \ell \in \mathbb{Z}\right\}$ tending to zero.

Note that (H2) implies the unique solvability of (2.1) (cf. [42, Thm. 4.11]). Moreover, we should remark that polygonal domains with an acute angle, whose both sides are nonparaxial, do not satisfy $(H 3)$.

We say that $\left\{\bar{\Omega}_{H}\right\}_{H \in \Lambda}$ is quasi-uniform if all possible quotients of mesh sizes of $\bar{\Omega}_{H}$ are bounded independently of $H$. Throughout this paper, we assume $H_{\max }$ is sufficiently small. By $W_{H}$, we denote the space of grid functions on $\bar{\Omega}_{H}$. For convenience, we tacitly assume that a function $v_{H} \in W_{H}$ is extended on $\mathbb{R}_{H}$ by zero. We often write $v_{P}$ instead of $v_{H}(P)$. For $P=\left(x_{j}, y_{\ell}\right) \in \bar{\Omega}_{H}$, let

$$
\square_{P}:=\left(x_{j-1 / 2}, x_{j+1 / 2}\right) \times\left(y_{\ell-1 / 2}, y_{\ell+1 / 2}\right) \cap \Omega, \quad \Gamma_{P}:=\left(x_{j-1 / 2}, x_{j+1 / 2}\right) \times\left(y_{\ell-1 / 2}, y_{\ell+1 / 2}\right) \cap \Gamma .
$$

Then

$$
\left(v_{H}, w_{H}\right)_{H}:=\sum_{P \in \bar{\Omega}_{H}}\left|\square_{P}\right| v_{P} \bar{w}_{P} \quad \text { and } \quad\left(\phi_{H}, \chi_{H}\right)_{\Gamma, H}:=\sum_{P \in \Gamma_{H}}\left|\Gamma_{P}\right| \phi_{P} \bar{\chi}_{P}
$$

defines an inner product on $W_{H}$ and on the space of grid functions on $\Gamma_{H}$, respectively. With the triangulation $\mathcal{T}_{H}$, we associate the piecewise linear interpolation $P_{H}: W_{H} \rightarrow \mathcal{C}(\bar{\Omega})$. 
Besides, we use the pointwise restriction $R_{H}: \mathcal{C}(\bar{\Omega}) \rightarrow W_{H}$ on the grid $\bar{\Omega}_{H}$ (or on $\Gamma_{H}$ for functions defined on $\Gamma$ ).

Let $A_{H}:=a_{H}+b_{H}+c_{H}+d_{H}+e_{H}+f_{H}+\alpha_{H}$ be the sesquilinear form defined by

$$
\begin{gathered}
a_{H}\left(v_{H}, w_{H}\right):=\sum_{\triangle \in \mathcal{T}_{H}} a_{\triangle, x} \int_{\triangle}\left(P_{H} v_{H}\right)_{x}\left(P_{H} \bar{w}_{H}\right)_{x} d V, \\
d_{H}\left(v_{H}, w_{H}\right):=\sum_{\triangle \in \mathcal{T}_{H}}\left(d P_{H} \bar{w}_{H}\right)_{\triangle, x} \int_{\triangle}\left(P_{H} v_{H}\right)_{x} d V, \\
f_{H}\left(v_{H}, w_{H}\right):=\left(R_{H} f v_{H}, w_{H}\right)_{H}, \quad \alpha_{H}\left(v_{H}, w_{H}\right):=\left(R_{H} \alpha v_{H}, w_{H}\right)_{\Gamma, H},
\end{gathered}
$$

and with forms $c_{H}$ and $e_{H}$ defined analogously to $a_{H}$ and $d_{H}$, respectively. Here, the subscript $\triangle, x$ denotes the value at the midpoint of the side of $\triangle \in \mathcal{T}_{H}$ parallel to the $x$-axis. The definition of the form $b_{H}$, which corresponds to mixed derivatives, is more intrigued and is based upon two special triangulations $\mathcal{T}_{H}^{(1)}$ and $\mathcal{T}_{H}^{(2)}$.

Let $\triangle_{j, \ell}^{(\perp)}$ denote an open triangle having an angle $\pi / 2$ at $\left(x_{j}, y_{\ell}\right) \in \mathbb{R}_{H}$ and two adjacent grid points as further vertices. We then define $\mathcal{T}_{H}^{(\nu)}:=\mathcal{T}_{H, 1}^{(\nu)} \cup \mathcal{T}_{H, 2}^{(\nu)}(\nu=1,2)$, where for $\nu=1,2$

$$
\begin{gathered}
\mathcal{T}_{H, 1}^{(\nu)}:=\left\{\triangle_{j, \ell}^{(\perp)} \subset \Omega:\left(x_{j}, y_{\ell}\right) \in \mathbb{R}_{H} \text { with } j+\ell+\nu \text { being even }\right\}, \\
\mathcal{T}_{H, 2}^{(\nu)}:=\left\{\triangle_{j, \ell}^{(\perp)} \subset\left(\Omega \backslash \bigcup_{\triangle \in \mathcal{T}_{H, 1}^{(\nu)}} \bar{\triangle}\right):\left(x_{j}, y_{\ell}\right) \in \mathbb{R}_{H} \text { with } j+\ell+\nu \text { being odd }\right\} .
\end{gathered}
$$

With $\mathcal{T}_{H}^{(\nu)}$, we associate the piecewise linear interpolation $P_{H}^{(\nu)}(\nu=1,2)$. Note that the triangulation $\mathcal{T}_{H}$ is in general a mixture of triangles from both $\mathcal{T}_{H}^{(1)}$ and $\mathcal{T}_{H}^{(2)}$, and that $\mathcal{T}_{H, 2}^{(\nu)}=\varnothing(\nu=1,2)$ if $\Omega$ is the union of rectangles. Moreover, let $\mathcal{T}_{H}^{\text {obl }} \subset \mathcal{T}_{H}$ be the set of triangles having one side on an oblique part of $\Gamma$.

Let $\left(x_{\triangle}, y_{\triangle}\right) \in \bar{\Omega}_{H}$ be the vertex of $\triangle \in \mathcal{T}_{H}$ that corresponds to the angle $\pi / 2$ and $\left(\tilde{x}_{\triangle}, y_{\triangle}\right),\left(x_{\triangle}, \tilde{y}_{\triangle}\right) \in \bar{\Omega}_{H}$ be the other two vertices. We then define

$$
b_{H}\left(v_{H}, w_{H}\right):=\frac{1}{2}\left(b_{H}^{(1)}\left(v_{H}, w_{H}\right)+b_{H}^{(2)}\left(v_{H}, w_{H}\right)\right)
$$

where for $\nu=1,2$

$$
b_{H}^{(\nu)}\left(v_{H}, w_{H}\right):=\sum_{\triangle \in \mathcal{T}_{H}^{(\nu)}} \int_{\triangle}\left(b_{\triangle, \tilde{x}}\left(P_{H}^{(\nu)} v_{H}\right)_{x}\left(P_{H}^{(\nu)} \bar{w}_{H}\right)_{y}+b_{\triangle, \tilde{y}}\left(P_{H}^{(\nu)} v_{H}\right)_{y}\left(P_{H}^{(\nu)} \bar{w}_{H}\right)_{x}\right) d V
$$

and

$$
b_{\triangle, \tilde{x}}:=\left\{\begin{array}{ll}
b\left(x_{\triangle}, y_{\triangle}\right) & \text { if } \triangle \in \mathcal{T}_{H, 1}^{(\nu)}, \\
b\left(\tilde{x}_{\triangle}, y_{\triangle}\right) & \text { if } \triangle \in \mathcal{T}_{H, 2}^{(\nu)},
\end{array} \quad b_{\triangle, \tilde{y}}:= \begin{cases}b\left(x_{\triangle}, y_{\triangle}\right) & \text { if } \Delta \in \mathcal{T}_{H, 1}^{(\nu)}, \\
b\left(x_{\triangle}, \tilde{y}_{\triangle}\right) & \text { if } \triangle \in \mathcal{T}_{H, 2}^{(\nu)} .\end{cases}\right.
$$

The fully discrete Galerkin approximation now reads as

find $u_{H} \in W_{H}$ such that $A_{H}\left(u_{H}, v_{H}\right)=\left(g_{H}, v_{H}\right)_{H}+\left(\psi_{H}, v_{H}\right)_{\Gamma, H}$ for all $v_{H} \in W_{H}$ (2.4) 
with the right-hand side $g_{H}$ and the boundary value $\psi_{H}$ defined via

$$
g_{P}:=\frac{1}{\left|\square_{P}\right|} \int_{\square_{P}} g d V \quad\left(P \in \bar{\Omega}_{H}\right), \quad \psi_{P}:=\psi(P) \quad\left(P \in \Gamma_{H}\right) .
$$

It can be shown that scheme (2.4) is equivalent to the finite difference approximation

$$
\begin{gathered}
A_{H} u_{H}:=-\delta_{x}^{(1 / 2)}\left(a \delta_{x}^{(1 / 2)} u_{H}\right)-\delta_{y}\left(b \delta_{x} u_{H}\right)-\delta_{x}\left(b \delta_{y} u_{H}\right)-\delta_{y}^{(1 / 2)}\left(c \delta_{y}^{(1 / 2)} u_{H}\right)+ \\
+R_{H} d \delta_{x} u_{H}+R_{H} e \delta_{y} u_{H}+R_{H} f u_{H}=g_{H} \quad \text { in } \Omega_{H},
\end{gathered}
$$

supplemented by an appropriate approximation of the boundary condition (cf. also [11]). In particular, we have

$$
A_{H}\left(v_{H}, w_{H}\right)=\left(A_{H} v_{H}, w_{H}\right)_{H}
$$

for all $v_{H}, w_{H} \in W_{H}$ with $w_{H}=0$ on $\Gamma_{H}$. Here, we make use of the divided differences

$$
\delta_{x}^{(1 / 2)} v_{j, \ell}:=\frac{v_{j+1 / 2, \ell}-v_{j-1 / 2, \ell}}{x_{j+1 / 2}-x_{j-1 / 2}}, \quad \delta_{x}^{(1 / 2)} v_{j+1 / 2, \ell}:=\frac{v_{j+1, \ell}-v_{j, \ell}}{x_{j+1}-x_{j}}, \quad \delta_{x} v_{j, \ell}:=\frac{v_{j+1, \ell}-v_{j-1, \ell}}{x_{j+1}-x_{j-1}}
$$

and the corresponding divided differences in the $y$-direction. We also apply these difference operators on functions defined on $\bar{\Omega}$ taking the points subscripted as arguments. In the case of mixed derivatives, the difference operator $A_{H}$ formally acts, near the oblique parts of $\Gamma$, on grid points outside $\bar{\Omega}_{H}$. The corresponding quantities are obtained in an antisymmetric way.

The proof of the following inverse stability property follows similar lines as that of $[12$, Thm. 2] and relies upon the discrete inf-sup condition for $A(\cdot, \cdot)$ (cf. [15, Thm. 8.2.8]).

Proposition 2.1. For $\bar{\Omega}_{H}(H \in \Lambda)$ with $H_{\max }$ being sufficiently small and $v_{H} \in W_{H}$, the following estimate holds true:

$$
\left\|P_{H} v_{H}\right\|_{1,2} \leqslant C \sup _{0 \neq w_{H} \in W_{H}} \frac{\left|A_{H}\left(v_{H}, w_{H}\right)\right|}{\left\|P_{H} w_{H}\right\|_{1,2}}
$$

By $C$, we denote a generic positive constant that is independent of significant quantities such as the grid size. As $\left\|P_{H} \cdot\right\|_{1,2}$ defines a norm on $W_{H}$, Proposition 2.1 shows the inverse stability of the difference operator $A_{H}:\left(W_{H},\left\|P_{H} \cdot\right\|_{1,2}\right) \rightarrow\left(W_{H},\left\|P_{H} \cdot\right\|_{1,2}\right)^{*}$. It also proves, for $H_{\max }$ being small enough, the unique solvability of the discrete problem even if the bilinear form is not strongly positive.

For the error of the numerical solution compared with the piecewise linear interpolant of the exact solution, we then find

$$
\left\|P_{H} R_{H} u-P_{H} u_{H}\right\|_{1,2} \leqslant C \sup _{0 \neq w_{H} \in W_{H}} \frac{\left|\tau_{H}\left(w_{H}\right)\right|}{\left\|P_{H} w_{H}\right\|_{1,2}}
$$

with the truncation error

$$
\tau_{H}\left(w_{H}\right):=A_{H}\left(R_{H} u, w_{H}\right)-A_{H}\left(u_{H}, w_{H}\right)=A_{H}\left(R_{H} u, w_{H}\right)-\left(g_{H}, w_{H}\right)_{H}-\left(\psi_{H}, w_{H}\right)_{\Gamma, H} .
$$

We can now state our main results that will be proved in the subsequent sections. 
Theorem 2.1. For $s \in(1 / 2,1]$, let $u \in H^{1+s}(\Omega)$, a, b, c, d, e, $f \in W^{1,2 /(1-s)}(\Omega), \alpha \in$ $W^{1,1 /(1-s)}(\Gamma), \psi \in H^{s}(\Gamma)$ and assume that $\left\{\bar{\Omega}_{H}\right\}_{H \in \Lambda}$ is quasi-uniform if $s \neq 1$. For $H_{\max }$ being sufficiently small, the discretisation error satisfies the estimate

$$
\begin{gathered}
\left\|P_{H} R_{H} u-P_{H} u_{H}\right\|_{1,2} \leqslant C\left(\sum_{P \in \bar{\Omega}_{H}}\left(\operatorname{diam} \square_{P}\right)^{2 s}\|u\|_{1+s, 2, \square_{P}}^{2}+\sum_{P \in \Gamma_{H}}\left|\Gamma_{P}\right|^{2 s}\left(\|u\|_{s, 2, \Gamma_{P}}^{2}+\|\psi\|_{s, 2, \Gamma_{P}}^{2}\right)\right)^{1 / 2} \leqslant \\
\leqslant C H_{\max }^{s}\left(\|u\|_{1+s, 2}+\|\psi\|_{s, 2, \Gamma}\right) .
\end{gathered}
$$

Remark 2.1. Theorem 2.1 remains true if the approximation $\psi_{H}=R_{H} \psi$ is replaced by an averaged approximation with $\psi_{P}=\left|\Gamma_{P}\right|^{-1} \int_{\Gamma_{P}} \psi d \sigma\left(P \in \Gamma_{H}\right)$.

If $\triangle \in \mathcal{T}_{H}$ has an edge on $\Gamma$, we denote it by $\Gamma_{P}$ with $P$ being its midpoint. For what follows, let $\Gamma_{1 / 2}$ be the set of all these midpoints and let $\Gamma_{1 / 2}^{x y} \subset \Gamma_{1 / 2}$ be the set of all midpoints corresponding to an oblique edge on $\Gamma$. The triangle corresponding to $P \in \Gamma_{1 / 2}^{x y}$ will be denoted by $\triangle_{P} \in \mathcal{T}_{H}^{\text {obl }}$. Moreover, we set $\sigma_{\text {obl }}:=1$ in the case of oblique boundary sections and $\sigma_{\mathrm{obl}}:=0$ otherwise, as well as $\sigma_{b}:=1$ if $b \neq 0$ and $\sigma_{b}:=0$ otherwise.

Theorem 2.2. For $s \in(1,2]$, let $u \in H^{1+s}(\Omega), a, b, c, d, e, f \in W^{2,2 /(2-s)}(\Omega), \alpha \in$ $W^{2,1 /(2-s)}(\Gamma), \psi \in H^{s}(\Gamma)$, and assume that $\left\{\bar{\Omega}_{H}\right\}_{H \in \Lambda}$ is quasi-uniform if $s \neq 2$. For $H_{\max }$ being sufficiently small, the discretisation error satisfies the estimate

$$
\begin{gathered}
\left\|P_{H} R_{H} u-P_{H} u_{H}\right\|_{1,2} \leqslant C\left(\sum_{P \in \bar{\Omega}_{H}}\left(\operatorname{diam} \square_{P}\right)^{2 s}\|u\|_{1+s, 2, \square_{P}}^{2}+\sum_{P \in \Gamma_{1 / 2}^{x y}}\left|\Gamma_{P}\right|^{2}\|u\|_{2,2, \triangle_{P}}^{2}+\right. \\
\left.+\sum_{P \in \Gamma_{1 / 2}}\left(\left(\sigma_{\mathrm{obl}}+\sigma_{b}\right)\left|\Gamma_{P}\right|^{\min (2 s, 3)}\|u\|_{1 / 2+s, 2, \Gamma_{P}}^{2}+\left|\Gamma_{P}\right|^{2 s}\left(\|u\|_{s, 2, \Gamma_{P}}^{2}+\|\psi\|_{s, 2, \Gamma_{P}}^{2}\right)\right)\right)^{1 / 2} \leqslant \\
\leqslant C H_{\max }^{s}\|\psi\|_{s, 2, \Gamma}+C\|u\|_{1+s, 2} \begin{cases}H_{\max }^{\min (s, 3 / 2)} & \text { if } s \neq 3 / 2, \\
H_{\max }^{3 / 2}\left|\log H_{\max }\right| & \text { if } s=3 / 2 .\end{cases}
\end{gathered}
$$

If $\Omega$ is the union of rectangles (i.e., $\Gamma_{1 / 2}^{x y}=\varnothing$ ), the local estimate above yields convergence of order $\mathcal{O}\left(H_{\max }^{\min (s, 3 / 2)}\right)$. If neither oblique boundary parts nor mixed derivatives appear, then the local estimate above implies order $\mathcal{O}\left(H_{\max }^{s}\right)$.

Remark 2.2. As one can infer from the proofs, the term $\sum_{P \in \Gamma_{1 / 2}^{x y}}\left|\Gamma_{P}\right|^{2}\|u\|_{2,2, \triangle_{P}}^{2}$ in the local estimate in Theorem 2.2 can be replaced by

$$
\sum_{P \in \Gamma_{1 / 2}^{x y}}\left(\left|\Gamma_{P}\right|^{3}\|u\|_{2,2, \triangle_{P}}^{2}+\sum_{S \subset \bar{\triangle}_{P}}\left|\Gamma_{P}\right|^{\min (2 s, 3)}\|u\|_{1 / 2+s, 2, S}^{2}\right)
$$

where $\sum_{S \subset \bar{\triangle}_{P}}$ is a finite sum over some characteristic line segments in $\bar{\triangle}_{P}$. If $u \in H^{1+s}(\Omega) \cap$ $W^{2, \infty}(\Omega)$, then $\sum_{P \in \Gamma_{1 / 2}^{x y}}\left|\Gamma_{P}\right|^{2}\|u\|_{2,2, \triangle_{P}}^{2}$ can also be replaced by $\sum_{P \in \Gamma_{1 / 2}^{x y}}\left(\left|\Gamma_{P}\right|^{3}\|u\|_{2,2, \triangle_{P}}^{2}+\right.$ $\left.\left|\Gamma_{P}\right|^{4}\|u\|_{2, \infty, \triangle_{P}}^{2}\right)$. In both cases, a global estimate of order $\mathcal{O}\left(H_{\max }^{\min (s, 3 / 2)}\right)$ without a logarithmic factor follows.

Interpolating the convergence results for $s=1$ and $s=2$ immediately proves 
Corollary 2.1. Let $s \in[1,2]$ and assume that $u \in H^{1+s}(\Omega), a, b, c, d, e, f \in W^{2, \infty}(\Omega)$, $\alpha \in W^{2, \infty}(\Gamma)$, and $\psi \in H^{s}(\Gamma)$. Moreover, let $\Omega$ be the union of rectangles. For $H_{\max }$ being sufficiently small, the discretisation error satisfies the estimate

$$
\left\|P_{H} R_{H} u-P_{H} u_{H}\right\|_{1,2} \leqslant C\left(\|u\|_{1+s, 2}+\|\psi\|_{s, 2, \Gamma}\right) \begin{cases}H_{\max }^{(1+s) / 2} & \text { if } b \neq 0 \\ H_{\max }^{s} & \text { if } b=0 .\end{cases}
$$

Although the preceding corollary provides convergence results for $s \in[1,2]$ even without assuming quasi-uniformity, neither local error estimates nor estimates for $s \in(1 / 2,1)$ can be derived in this way.

Remark 2.3. In the case of no mixed derivatives or no oblique boundary sections, the truncation error $\tau_{H}\left(w_{H}\right)$ is of optimal order $\mathcal{O}\left(H_{\max }^{s}\right)$ if $w_{H}$ vanishes on $\Gamma$, for $s=2$ even without assuming quasi-uniformity. If, however, mixed derivatives and oblique boundary sections occur, then for $s \geqslant 3 / 2$ only suboptimal estimates can be derived; for $s=2$ and $w_{H}=0$ on $\Gamma$, the suboptimal estimate remains valid again without assuming quasiuniformity. This is in accordance with the results known from [13] for the Dirichlet problem with mixed derivatives in a domain with oblique boundary sections where an error estimate of order $\mathcal{O}\left(H_{\max }^{3 / 2-\varepsilon}\right)$ with $\varepsilon>0$ has been shown if $u \in H^{3}(\Omega)$.

Remark 2.4. As is shown in [11, Remark 5.3, 5.4], the averaged restriction of the righthand side $g$ in (2.5) can be replaced by the pointwise restriction on $\bar{\Omega}_{H}$ if $g \in H^{s}(\Omega)$ for $s \in(1,2]$ retaining the order of convergence. If, however, $g$ is not smooth enough, then working with the pointwise restriction may destroy the higher order.

\section{Notation}

In this section, we collect all the notation that will be used in the course of proving the main results. Always, definitions analogous to those for the $x$-direction apply for the $y$-direction and vice versa. We firstly define some sets of points:

$$
\begin{gathered}
\Omega_{1 / 2}^{x}:=\left\{\left(x_{j+1 / 2}, y_{\ell}\right) \in \bar{\Omega}: j, \ell \in \mathbb{Z}\right\}, \quad \Omega_{1 / 2}^{x y}:=\left\{\left(x_{j+1 / 2}, y_{\ell+1 / 2}\right) \in \bar{\Omega}: j, \ell \in \mathbb{Z}\right\}, \\
\Gamma_{1 / 2}^{x}:=\Omega_{1 / 2}^{x} \cap \Gamma, \quad \Gamma_{1 / 2}^{x y}:=\Omega_{1 / 2}^{x y} \cap \Gamma, \quad \Gamma_{1 / 2}:=\Gamma_{1 / 2}^{x} \cup \Gamma_{1 / 2}^{y} \cup \Gamma_{1 / 2}^{x y} .
\end{gathered}
$$

Let $P=\left(x_{j+1 / 2}, y_{\ell}\right) \in \Omega_{1 / 2}^{x}$. Then we set

$$
\square_{P}:=\left(x_{j}, x_{j+1}\right) \times\left(y_{\ell-1 / 2}, y_{\ell+1 / 2}\right) \cap \Omega, \quad h_{P}:=h_{j}, \quad S_{P}:=\left\{x_{j+1 / 2}\right\} \times\left(y_{\ell-1 / 2}, y_{\ell+1 / 2}\right) \cap \Omega
$$

as well as $P^{l}:=\left(x_{j}, y_{\ell}\right), P^{r}:=\left(x_{j+1}, y_{\ell}\right)$, and $v_{P^{r l}}:=\left(v\left(P^{r}\right)+v\left(P^{l}\right)\right) / 2$ for the function $v$.

For $P=\left(x_{j}, y_{\ell+1 / 2}\right) \in \Omega_{1 / 2}^{y}$ lying not on $\Gamma$ or a side of a triangle $\triangle \in \mathcal{T}_{H}^{\text {obl }}$, we set

$$
\begin{gathered}
P^{1}:=\left(x_{j+1 / 2}, y_{l+1}\right), \quad P^{2}:=\left(x_{j-1 / 2}, y_{l+1}\right), \quad P^{3}:=\left(x_{j-1 / 2}, y_{l}\right), \quad P^{4}:=\left(x_{j+1 / 2}, y_{\ell}\right), \\
P^{l}:=\left(x_{j-1 / 2}, y_{\ell+1 / 2}\right), \quad P^{r}:=\left(x_{j+1 / 2}, y_{\ell+1 / 2}\right), \quad \bar{P}:=\left(x_{j-1 / 2}+h_{P} / 2, y_{\ell+1 / 2}\right),
\end{gathered}
$$

with $h_{P}:=x_{j+1 / 2}-x_{j-1 / 2}=\left(h_{j-1}+h_{j}\right) / 2$ and

$$
\begin{gathered}
S_{P}^{1}:=\left\{x_{j+1 / 2}\right\} \times\left(y_{\ell+1 / 2}, y_{\ell+1}\right), \quad S_{P}^{2}:=\left\{x_{j-1 / 2}\right\} \times\left(y_{\ell+1 / 2}, y_{\ell+1}\right), \\
S_{P}^{3}:=\left\{x_{j-1 / 2}\right\} \times\left(y_{\ell}, y_{\ell+1 / 2}\right), \quad S_{P}^{4}:=\left\{x_{j+1 / 2}\right\} \times\left(y_{\ell}, y_{\ell+1 / 2}\right) .
\end{gathered}
$$


The same definitions apply for $P \in \Gamma_{1 / 2}^{y}$ or a point $P \in \Omega_{1 / 2}^{y}$ that lies on a side of a triangle $\triangle \in \mathcal{T}_{H}^{\text {obl }}$ but with $x_{j+1 / 2}$ and $x_{j-1 / 2}$ being replaced by $x_{j}, h_{P}$ being replaced by $h_{j}$ and $h_{j-1}$ if $\left(x_{j+1}, y_{\ell+1 / 2}\right)$ and $\left(x_{j-1}, y_{\ell+1 / 2}\right)$ lies outside $\bar{\Omega}$, respectively (see also Figure).
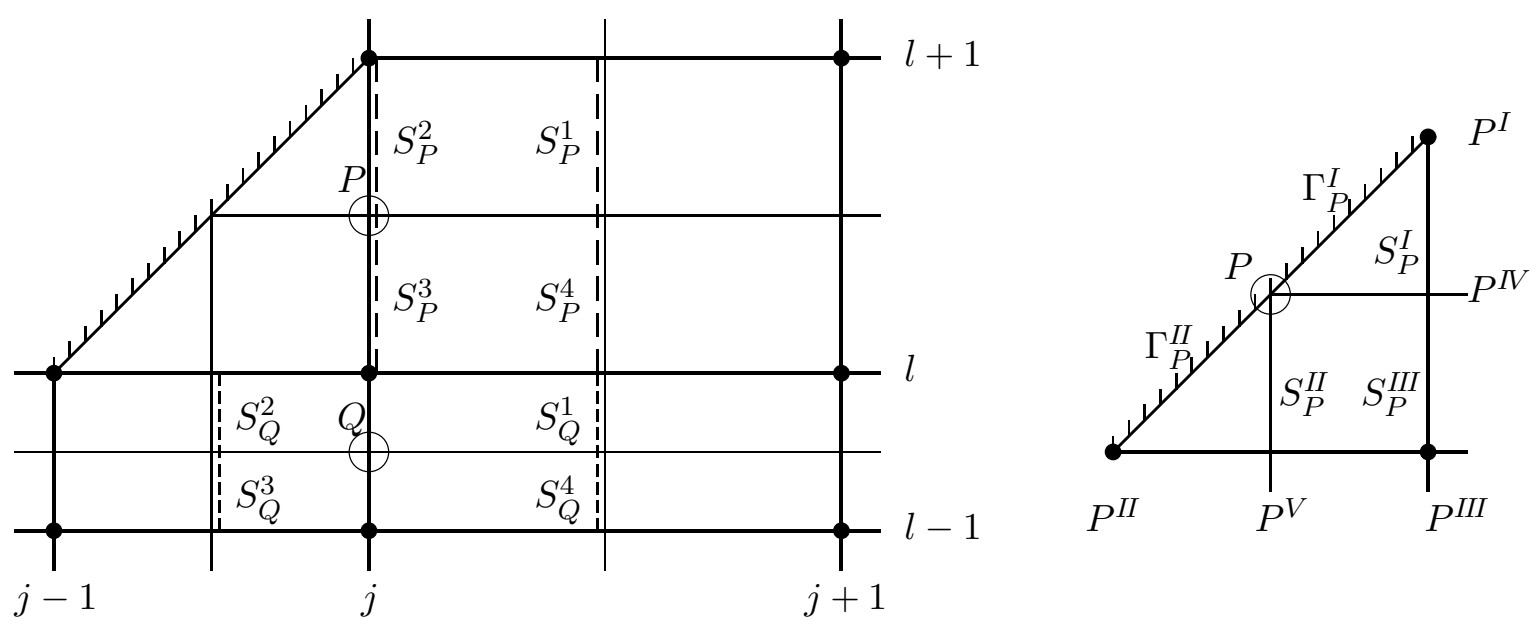

Notation for $P, Q \in \Omega_{1 / 2}^{y}$ (left) and triangle $\triangle \in \mathcal{T}_{H}^{\text {obl }}$

For $P=\left(x_{j+1 / 2}, y_{\ell+1 / 2}\right) \in \Omega_{1 / 2}^{x y}$, we define

$\square_{P}:=\left(x_{j}, x_{j+1}\right) \times\left(y_{\ell}, y_{\ell+1}\right) \cap \Omega, \quad h_{P}:=h_{j}, k_{P}:=k_{\ell}, \quad S_{P}:=\left\{x_{j+1 / 2}\right\} \times\left(y_{\ell}, y_{\ell+1}\right) \cap \Omega$,

$$
S_{P}^{-}:=\left\{x_{j+1 / 2}\right\} \times\left(y_{\ell}, y_{\ell+1 / 2}\right) \cap \Omega, \quad S_{P}^{+}:=\left\{x_{j+1 / 2}\right\} \times\left(y_{\ell+1 / 2}, y_{\ell+1}\right) \cap \Omega,
$$

and

$$
\begin{gathered}
P^{1}:=\left(x_{j+1}, y_{\ell+1}\right), \quad P^{2}:=\left(x_{j}, y_{\ell+1}\right), \quad P^{3}:=\left(x_{j}, y_{\ell}\right), \quad P^{4}:=\left(x_{j+1}, y_{\ell}\right), \\
P^{-}:=\left(x_{j+1 / 2}, y_{\ell}\right), \quad P^{+}:=\left(x_{j+1 / 2}, y_{\ell+1}\right), \quad P^{l}:=\left(x_{j}, y_{\ell+1 / 2}\right), \quad P^{r}:=\left(x_{j+1}, y_{\ell+1 / 2}\right) .
\end{gathered}
$$

For $P \in \Omega_{1 / 2}^{x y} \backslash \Gamma$, the rectangle $\square_{P}$ can be split into four congruent rectangles $\square_{P}^{(i)}$ having $P^{(i)}$ $(i=1, \ldots, 4)$ as one vertex. For the function $v$, we again define $v_{P^{r l}}:=\left(v\left(P^{r}\right)+v\left(P^{l}\right)\right) / 2$.

For $P=\left(x_{j}, y_{\ell+1 / 2}\right) \in \Gamma_{1 / 2}^{y}$, let $P^{I}:=\left(x_{j}, y_{\ell+1}\right), P^{I I}:=\left(x_{j}, y_{\ell}\right)$, and

$$
\Gamma_{P}:=\left\{x_{j}\right\} \times\left(y_{\ell}, y_{\ell+1}\right), \quad \Gamma_{P}^{I}:=\left\{x_{j}\right\} \times\left(y_{\ell+1 / 2}, y_{\ell+1}\right), \quad \Gamma_{P}^{I I}:=\left\{x_{j}\right\} \times\left(y_{\ell}, y_{\ell+1 / 2}\right) .
$$

A triangle $\triangle \in \mathcal{T}_{H}^{\text {obl }}$ can be characterised by the point $P=\left(x_{j+1 / 2}, y_{\ell+1 / 2}\right) \in \Gamma_{1 / 2}^{x y}$ lying on its oblique side, and we often write $\triangle_{P}$ instead of $\square_{P}$. We set $h_{P}:=h_{j}, k_{P}:=k_{\ell}$. Moreover, let $P^{I}, P^{I I}, P^{I I I}$ be the vertices of this triangle such that $P^{I I I}$ always corresponds to the angle $\pi / 2$, the line between $P^{I}$ and $P^{I I I}$ is parallel to the $y$-axis, and the line between $P^{I I}$ and $P^{I I I}$ is parallel to the $x$-axis. By $P^{I V}$ and $P^{V}$, we denote the midpoint of the side parallel to the $y$ - and $x$-axis, respectively. We have $\Gamma_{P}=\Gamma_{P}^{I} \cup \Gamma_{P}^{I I}$ with $\Gamma_{P}^{i}$ being the line segment between $P$ and $P^{i}(i \in\{I, I I\})$. The line segment between $P^{I V}$ and $P^{I}$ is denoted by $S_{P}^{I}$, the one between $P^{V}$ and $P$ by $S_{P}^{I I}$, and the one between $P^{I I I}$ and $P^{I V}$ by $S_{P}^{I I I}$ (see Figure). Furthermore, the triangle $\triangle_{P}$ can be split into a triangle $\triangle_{P}^{I}$ with vertices $P^{I}, P, P^{I V}$, a triangle $\triangle_{P}^{I I}$ with vertices $P^{I I}, P^{V}, P$, and a rectangle $\square_{P}^{I I I}$ with vertices $P^{I I I}, P^{I V}, P, P^{V}$. Note that $\left|\triangle_{P}^{I}\right|=\left|\triangle_{P}^{I I}\right|=\left|\square_{P}^{I I I}\right| / 2=\left|\triangle_{P}\right| / 4$.

Finally, for a point $P$ whatsoever, we denote by $x_{P}$ and $y_{P}$ its coordinates. 


\section{Low-order convergence: proof of Theorem 2.1}

The truncation error (2.7) can be decomposed in terms corresponding to the parts of the differential operator. For all $w_{H} \in W_{H}$, we find from $(2.3)-(2.5)$ the definitions of $A$, $A_{H}(\cdot, \cdot)$, and the Green formula

$$
\begin{gathered}
\tau_{H}\left(w_{H}\right)=A_{H}\left(R_{H} u, w_{H}\right)-\sum_{P \in \bar{\Omega}_{H}} \int_{\square_{P}} A u d V \bar{w}_{P}-\left(\psi_{H}, w_{H}\right)_{\Gamma, H}:= \\
:=\tau_{H}^{(a)}\left(w_{H}\right)+\tau_{H}^{(b)}\left(w_{H}\right)+\tau_{H}^{(c)}\left(w_{H}\right)+\tau_{H}^{(d)}\left(w_{H}\right)+\tau_{H}^{(e)}\left(w_{H}\right)+\tau_{H}^{(f)}\left(w_{H}\right)+\tau_{\Gamma, H}\left(w_{H}\right),
\end{gathered}
$$

where

$$
\begin{gathered}
\tau_{H}^{(a)}\left(w_{H}\right):=a_{H}\left(R_{H} u, w_{H}\right)-\sum_{P \in \Omega_{1 / 2}^{x}} h_{P} \int_{S_{P}} a u_{x} d y \delta_{x}^{(1 / 2)} \bar{w}_{P}, \\
\tau_{H}^{(d)}\left(w_{H}\right):=d_{H}\left(R_{H} u, w_{H}\right)-\sum_{P \in \bar{\Omega}_{H} \square_{P}} d u_{x} d V \bar{w}_{P}, \\
\tau_{H}^{(f)}\left(w_{H}\right):=\sum_{P \in \bar{\Omega}_{H}}\left|\square_{P}\right|\left((f u)(P)-\frac{1}{\left|\square_{P}\right|} \iint_{\square_{P}} f u d V\right) \bar{w}_{P}, \\
\tau_{\Gamma, H}\left(w_{H}\right):=\sum_{P \in \Gamma_{H}}\left(\int_{\Gamma_{P}}(\psi-\alpha u) d \sigma-\left|\Gamma_{P}\right|(\psi-\alpha u)(P)\right) \bar{w}_{P},
\end{gathered}
$$

and $\tau_{H}^{(b)}, \tau_{H}^{(c)}, \tau_{H}^{(e)}$ are given analogously.

The following result is already known from [11] for a domain that is the union of rectangles. The proof in the present case of $\Omega$ having oblique boundary sections follows the same lines as that of $[11$, Lemmata $4.2,4.3,4.4]$ and is omitted here.

Lemma 4.1. Let $s \in(1 / 2,1], u \in H^{1+s}(\Omega), a \in W^{1,2 /(1-s)}(\Omega)$ and assume that $\left\{\bar{\Omega}_{H}\right\}_{H \in \Lambda}$ is quasi-uniform if $s \neq 1$. Then for all $w_{H} \in W_{H}$

$$
\left|\tau_{H}^{(a)}\left(w_{H}\right)\right| \leqslant C\left(\sum_{P \in \Omega_{1 / 2}^{x}}\left(\operatorname{diam} \square_{P}\right)^{2 s}\|u\|_{1+s, 2, \square_{P}}^{2}\right)^{1 / 2}\left\|P_{H} w_{H}\right\|_{1,2} .
$$

For the mixed derivatives, we observe the following.

Lemma 4.2. Let $s \in(1 / 2,1], u \in H^{1+s}(\Omega), b \in W^{1,2 /(1-s)}(\Omega)$ and assume that $\left\{\bar{\Omega}_{H}\right\}_{H \in \Lambda}$ is quasi-uniform if $s \neq 1$. Then for all $w_{H} \in W_{H}$

$$
\left|\tau_{H}^{(b)}\left(w_{H}\right)\right| \leqslant C\left(\sum_{P \in \Omega_{1 / 2}^{x y}}\left(\operatorname{diam} \square_{P}\right)^{2 s}\|u\|_{1+s, 2, \square_{P}}^{2}\right)^{1 / 2}\left\|P_{H} w_{H}\right\|_{1,2} .
$$

Proof. In what follows, we decompose $\tau_{H}^{(b)}$ into the part $\tau_{H}^{(b, x y)}$ corresponding to $-\left(b u_{x}\right)_{y}$ plus the part $\tau_{H}^{(b, y x)}$ corresponding to $-\left(b u_{y}\right)_{x}$ focussing only on the latter as the former can be handled similarly. Analogously, we decompose $b_{H}\left(R_{H} u, w_{H}\right)=b_{H}^{x y}\left(R_{H} u, w_{H}\right)+$ $b_{H}^{y x}\left(R_{H} u, w_{H}\right)$. With integration and summation by parts, we find

$$
\tau_{H}^{(b, y x)}\left(w_{H}\right)=b_{H}^{y x}\left(R_{H} u, w_{H}\right)-\sum_{P \in \Omega_{1 / 2}^{x}} h_{P} \int_{S_{P}} b u_{y} d y \delta_{x}^{(1 / 2)} \bar{w}_{P}=b_{H}^{y x}\left(R_{H} u, w_{H}\right)-
$$


$-\sum_{P \in \Omega_{1 / 2}^{x y} \backslash \Gamma_{1 / 2}^{x y}} h_{P}\left(\int_{S_{P}^{-}} b u_{y} d y \delta_{x}^{(1 / 2)} \bar{w}_{P^{-}}+\int_{S_{P}^{+}} b u_{y} d y \delta_{x}^{(1 / 2)} \bar{w}_{P^{+}}\right)-\sum_{P \in \Gamma_{1 / 2}^{x y}} \operatorname{sgn} \eta_{x}(P) \int_{S_{P}^{I I}} b u_{y} d y\left(\bar{w}_{P^{I I}}-\bar{w}_{P I I I}\right)$.

The definition of $b_{H}$ leads to

$$
\begin{aligned}
b_{H}^{y x}\left(R_{H} u, w_{H}\right)= & \frac{1}{4} \sum_{P \in \Omega_{1 / 2}^{x y} \backslash \Gamma_{1 / 2}^{x y}}\left|\square_{P}\right|\left(b\left(P^{1}\right) \delta_{y}^{(1 / 2)} u_{P^{r}} \delta_{x}^{(1 / 2)} \bar{w}_{P^{+}}+b\left(P^{2}\right) \delta_{y}^{(1 / 2)} u_{P^{l}} \delta_{x}^{(1 / 2)} \bar{w}_{P^{+}}+\right. \\
& \left.+b\left(P^{3}\right) \delta_{y}^{(1 / 2)} u_{P^{l}} \delta_{x}^{(1 / 2)} \bar{w}_{P^{-}}+b\left(P^{4}\right) \delta_{y}^{(1 / 2)} u_{P^{r}} \delta_{x}^{(1 / 2)} \bar{w}_{P^{-}}\right)+ \\
+ & \frac{1}{4} \sum_{P \in \Gamma_{1 / 2}^{x y}} \operatorname{sgn} \eta_{x}(P) k_{P}\left(b\left(P^{I}\right)+b\left(P^{I I I}\right)\right) \delta_{y}^{(1 / 2)} u_{P^{I V}}\left(\bar{w}_{P^{I I}}-\bar{w}_{P^{I I I}}\right)
\end{aligned}
$$

We firstly concentrate on the contributions corresponding to $\Omega_{1 / 2}^{x y} \backslash \Gamma_{1 / 2}^{x y}$. We find

$$
\delta_{y}^{(1 / 2)} u_{P^{l}}=\frac{1}{k_{P}} \int_{y_{P}-k_{P} / 2}^{y_{P}+k_{P} / 2} u_{y}\left(x_{P}-\frac{h_{P}}{2}, y\right) d y, \quad P \in \Omega_{1 / 2}^{x y}
$$

and an analogous identity holds with $P^{r}$ instead of $P^{l}$. Transformation on a reference square $\hat{\square}$, employing the continuous embedding $H^{s}(\hat{\square}) \hookrightarrow L^{1}(\hat{S})$ with an arbitrary vertical line segment $\hat{S} \subset \hat{\square}$, and rescaling gives

$$
\left|\delta_{y}^{(1 / 2)} u_{P^{l}}\right| \leqslant C\left|\square_{P}\right|^{-1 / 2}\left\|u_{y}\right\|_{s, 2, \square_{P}}
$$

and the same estimate with $P^{r}$ instead of $P^{l}$. Since $b \in W^{1,2 /(1-s)}(\Omega) \hookrightarrow \mathcal{C}(\bar{\Omega})$, the generalised Bramble-Hilbert lemma (cf. [10]) and the usual scaling and rescaling now yields for $s \in(1 / 2,1], P \in \Omega_{1 / 2}^{x y}, i \in\{1,2,3,4\}$, and $Q \in\left\{P^{r}, P^{l}\right\}$

$$
\begin{gathered}
\left|\square_{P}\right|^{1 / 2}\left|b\left(P^{i}\right)-b(P)\right|\left|\delta_{y}^{(1 / 2)} u_{Q}\right| \leqslant C\left|\square_{P}\right|^{-(1-s) / 2} \max \left(h_{P}, k_{P}\right)|b|_{1,2 /(1-s)}\left\|u_{y}\right\|_{s, 2, \square_{P}} \leqslant \\
\leqslant C \max \left(h_{P}, k_{P}\right)^{s}\left\|u_{y}\right\|_{s, 2, \square_{P}} .
\end{gathered}
$$

Here, we have employed $\left|\square_{P}\right|^{-(1-s) / 2} \max \left(h_{P}, k_{P}\right) \leqslant C \max \left(h_{P}, k_{P}\right)^{s}$ which requires quasiuniformity for $s \in(1 / 2,1)$ but not for $s=1$. The preceding estimate together with the Cauchy-Schwarz inequality and

$$
\sum_{P \in \Omega_{1 / 2}^{x y}}\left|\square_{P}\right|\left(\left|\delta_{x}^{(1 / 2)} w_{P^{-}}\right|^{2}+\left|\delta_{x}^{(1 / 2)} w_{P^{+}}\right|^{2}\right) \leqslant C\left|P_{H} w_{H}\right|_{1,2}^{2}
$$

shows that replacing $b\left(P^{i}\right)$ by $b(P)$ in (4.7) leads to an error that is bounded as desired.

Since $\delta_{y}^{(1 / 2)} u_{P^{l}}+\delta_{y}^{(1 / 2)} u_{P^{r}}-4\left(u\left(P^{+}\right)-u(P)\right) / k_{P}$ vanishes for $u=1, x, y$ and since $u \in$ $H^{1+s}(\Omega) \hookrightarrow \mathrm{C}(\bar{\Omega})$, we find again with the generalised Bramble-Hilbert lemma for $s<1$

$$
\left|\square_{P}\right|^{1 / 2}|b(P)|\left|\delta_{y}^{(1 / 2)} u_{P^{l}}+\delta_{y}^{(1 / 2)} u_{P^{r}}-\frac{4}{k_{P}}\left(u\left(P^{+}\right)-u(P)\right)\right| \leqslant C \max \left(h_{P}, k_{P}\right)^{s}|u|_{1+s, 2, \square_{P}} .
$$

The same estimate applies with $u(P)-u\left(P^{-}\right)$instead of $u\left(P^{+}\right)-u(P)$. 
If $s=1$, we observe that for $P=\left(x_{j+1 / 2}, y_{\ell+1 / 2}\right) \in \Omega_{1 / 2}^{x y}$

$\delta_{y}^{(1 / 2)} u_{P^{l}}+\delta_{y}^{(1 / 2)} u_{P^{r}}-\frac{4}{k_{P}}\left(u\left(P^{+}\right)-u(P)\right)=\frac{1}{k_{\ell}} \int_{y_{\ell}}^{y_{\ell+1}}\left(u_{y}\left(x_{j}, y\right)+u_{y}\left(x_{j+1}, y\right) d y-\frac{4}{k_{\ell}} \int_{y_{\ell+1 / 2}}^{y_{\ell+1}} u_{y}\left(x_{j+1 / 2}, y\right) d y\right.$

vanishes for $u_{y}=1$. The Bramble - Hilbert lemma furnishes the estimate wanted without assuming quasi-uniformity which remains true with $u(P)-u\left(P^{-}\right)$instead of $u\left(P^{+}\right)-u(P)$.

With $\left|\square_{P}\right|=h_{P} k_{P}$ and after applying the triangle inequality, the following contribution is left from (4.6) and (4.7) with the following contribution:

$$
\begin{gathered}
\sum_{P \in \Omega_{1 / 2}^{x y} \backslash \Gamma_{1 / 2}^{x y}} h_{P}\left(\left(b(P)\left(u\left(P^{+}\right)-u(P)\right)-\int_{S_{P}^{+}} b u_{y} d y\right) \delta_{x}^{(1 / 2)} \bar{w}_{P^{+}}+\left(b(P)\left(u(P)-u\left(P^{-}\right)\right)-\right.\right. \\
\left.\left.-\int_{S_{P}^{-}} b u_{y} d y\right) \delta_{x}^{(1 / 2)} \bar{w}_{P^{-}}\right)=\sum_{P \in \Omega_{1 / 2}^{x y}} h_{P}\left(\int_{S_{P}^{+}}(b(P)-b) u_{y} d y \delta_{x}^{(1 / 2)} \bar{w}_{P^{+}}+\int_{S_{P}^{-}}(b(P)-b) u_{y} d y \delta_{x}^{(1 / 2)} \bar{w}_{P^{-}}\right) .
\end{gathered}
$$

Similar to the arguments used before, we have

$$
h_{P}^{1 / 2} k_{P}^{-1 / 2}\left|\int_{S_{P}^{+}}(b(P)-b) u_{y} d y\right| \leqslant C \max \left(h_{P}, k_{P}\right)^{s}|b|_{1,2 /(1-s)}\left\|u_{y}\right\|_{s, 2, \square_{P}}
$$

and the same bound applies for the term corresponding to $S_{P}^{-}$. For $s=1$, the estimate holds again without assuming quasi-uniformity. Together with the Cauchy - Schwarz inequality, the asserted bound follows.

It remains to estimate the contributions to (4.6) and (4.7) arising from $\Gamma_{1 / 2}^{x y}$, i.e.,

$$
G_{1}:=\sum_{P \in \Gamma_{1 / 2}^{x y}} \operatorname{sgn} \eta_{x}(P)\left(\frac{k_{P}}{4}\left(b\left(P^{I}\right)+b\left(P^{I I I}\right)\right) \delta_{y}^{(1 / 2)} u_{P^{I V}}-\int_{S_{P}^{I I}} b u_{y} d y\right)\left(\bar{w}_{P I I}-\bar{w}_{P I I I}\right) .
$$

Firstly, we see that $k_{P} \delta_{y}^{(1 / 2)} u_{P N}-2 \int_{S_{P}^{I I}} u_{y} d y$ vanishes for $u=1, x, y$ and is linear and bounded for $u \in H^{1+s}(\Omega)$. The generalised Bramble-Hilbert lemma, together with

$$
\left|w_{P I I}-w_{P I I I}\right| \leqslant C h_{P}^{1 / 2} k_{P}^{-1 / 2}\left|P_{H} w_{H}\right|_{1,2, \triangle_{P}}
$$

$b \in W^{1,2 /(1-s)}(\Omega) \hookrightarrow \mathcal{C}(\bar{\Omega})$, and the Cauchy-Schwarz inequality, thus shows that $k_{P} \delta_{y}^{(1 / 2)} u_{P}{ }^{I V}$ in (4.10) can be replaced by $2 \int_{S_{P}^{I I}} u_{y} d y$ as the error can be bounded appropriately. Here, quasi-uniformity has not to be assumed if $s=1$.

Secondly, we observe that $\left(b\left(P^{I}\right)+b\left(P^{I I I}\right)\right) \int_{S_{P}^{I I}} u_{y} d y-2 \int_{S_{P}^{I I}} b u_{y} d y$ vanishes for $b=1$ and is linear and bounded for $b \in W^{1,2 /(1-s)}(\Omega), u_{y} \in H^{s}(\Omega) \hookrightarrow L^{1}(\Omega)$. The Bramble-Hilbert lemma again yields the optimal estimate that is valid for $s=1$ even without assuming quasi-uniformity.

We now come to the first-order terms. 
Lemma 4.3. Let $s \in(1 / 2,1], u \in H^{1+s}(\Omega), d \in W^{1,2 /(1-s)}(\Omega)$, and assume that $\left\{\bar{\Omega}_{H}\right\}_{H \in \Lambda}$ is quasi-uniform if $s \neq 1$. Then for all $w_{H} \in W_{H}$

$$
\left|\tau_{H}^{(d)}\left(w_{H}\right)\right| \leqslant C\left(\sum_{P \in \bar{\Omega}_{H}}\left(\operatorname{diam} \square_{P}\right)^{2 s}\|u\|_{1+s, 2, \square_{P}}^{2}\right)^{1 / 2}\left\|P_{H} w_{H}\right\|_{1,2} .
$$

Proof. A simple calculation shows that

$$
d_{H}\left(R_{H} u, w_{H}\right)=\sum_{P \in \Omega_{1 / 2}^{x}} h_{P}\left|S_{P}\right| d(P) \delta_{x}^{(1 / 2)} u_{P}\left(P_{H} \bar{w}_{H}\right)(P),
$$

where $\left(P_{H} \bar{w}_{H}\right)(P)=\left(\bar{w}_{j, \ell}+\bar{w}_{j+1, \ell}\right) / 2$ for $P=\left(x_{j+1 / 2}, y_{\ell}\right)$. Note that $h_{P}\left|S_{P}\right| \neq\left|\square_{P}\right|$ if $P \in \Omega_{1 / 2}^{x}$ lies on an edge of a triangle $\triangle \in \mathcal{T}_{H}^{\text {obl }}$. For $P \in \Omega_{1 / 2}^{x}$, we set

$$
\square_{P}^{\prime}:=\square_{P} \backslash \bigcup_{\triangle \in \mathcal{T}_{H}^{\text {obl }}} \bar{\triangle}, \quad S_{P}^{\prime}:=S_{P} \backslash \bigcup_{\triangle \in \mathcal{T}_{H}^{\text {obl }}} \bar{\triangle},
$$

which allows to consider the contributions from triangles $\triangle \in \mathcal{T}_{H}^{\text {obl }}$ separately. The definition of $\square_{P}^{\prime}$ also applies for points $P \in \bar{\Omega}_{H}$. We then have with (4.3) that

$$
\tau_{H}^{(d)}\left(w_{H}\right)=F_{1}+F_{2}+F_{3}+G
$$

where

$$
\begin{gathered}
F_{1}:=\sum_{P \in \Omega_{1 / 2}^{x}}\left|\square_{P}^{\prime}\right| d(P)\left(\delta_{x}^{(1 / 2)} u_{P}-\left|\square_{P}^{\prime}\right|^{-1} \int_{\square_{P}^{\prime}} u_{x} d V\right)\left(P_{H} \bar{w}_{H}\right)(P), \\
F_{2}:=\sum_{P \in \Omega_{1 / 2}^{x} \square_{P}^{\prime}} \int_{P}(d(P)-d) u_{x} d V\left(P_{H} \bar{w}_{H}\right)(P), \quad F_{3}:=\sum_{P \in \Omega_{1 / 2}^{x} \square_{P}^{\prime}} \int_{\square^{\prime}} d u_{x} d V\left(P_{H} \bar{w}_{H}\right)(P)-\sum_{P \in \bar{\Omega}_{H} \square_{P}^{\prime}} d u_{x} d V \bar{w}_{P}, \\
G:=\sum_{P \in \Gamma_{1 / 2}^{x y}}\left(\left|\triangle_{P}\right| d\left(P^{V}\right) \delta_{x}^{(1 / 2)} u_{P V}\left(P_{H} \bar{w}_{H}\right)\left(P^{V}\right)-\int_{\triangle_{P}^{I}} d u_{x} d V \bar{w}_{P^{I}}-\int_{\triangle_{P}^{I I}} d u_{x} d V \bar{w}_{P}^{I I}-\int_{\square_{P}^{I I I}} d u_{x} d V \bar{w}_{P I I}\right) .
\end{gathered}
$$

Since $\delta_{x}^{(1 / 2)} u_{P}-\left|\square_{P}^{\prime}\right|^{-1} \int_{\square_{P}^{\prime}} u_{x} d V\left(P \in \Omega_{1 / 2}^{x}\right)$ vanishes for $u=1, x, y$ and since $H^{1+s}(\Omega) \hookrightarrow$ $\mathrm{C}(\bar{\Omega}), H^{1+s}(\Omega) \hookrightarrow W^{1,1}(\Omega)$, we find for $s \in(1 / 2,1)$ with the generalised Bramble-Hilbert lemma

$$
\left|F_{1}\right| \leqslant C\left(\sum_{P \in \Omega_{1 / 2}^{x}}\left|\square_{P}^{\prime}\right|^{-1} h_{P}^{-2} \max \left(h_{P},\left|S_{P}^{\prime}\right|\right)^{2(2+s)}|u|_{1+s, 2, \square_{P}}^{2}\right)^{1 / 2}\left\|P_{H} w_{H}\right\|_{0,2} .
$$

Here, we have used the fact that $d \in W^{1,2 /(1-s)}(\Omega) \hookrightarrow \mathcal{C}(\bar{\Omega})$ and

$$
\sum_{P \in \Omega_{1 / 2}^{x}}\left|\square_{P}^{\prime}\right|\left|\left(P_{H} w_{H}\right)(P)\right|^{2} \leqslant C\left\|P_{H} w_{H}\right\|_{0,2}^{2}
$$

Because of the quasi-uniformity of $\left\{\bar{\Omega}_{H}\right\}_{H \in \Lambda}$, we have $\left|\square_{P}^{\prime}\right|^{-1} h_{P}^{-2} \max \left(h_{P},\left|S_{P}^{\prime}\right|\right)^{4} \leqslant C$, and the estimate wanted follows. If $s=1$, we observe that for $P \in \Omega_{1 / 2}^{x}$

$$
\left|\square_{P}^{\prime}\right|^{1 / 2}\left|\delta_{x}^{(1 / 2)} u_{P}-\frac{1}{\left|\square_{P}^{\prime}\right|} \int_{\square_{P}^{\prime}} u_{x} d V\right|=\frac{1}{\left|\square_{P}^{\prime}\right|^{1 / 2}}\left|\int_{\square_{P}^{\prime}}\left(u_{x}\left(x, y_{P}\right)-u_{x}(x, y)\right) d V\right| \leqslant\left|S_{P}^{\prime}\right|\left\|u_{x y}\right\|_{0,2, \square_{P}^{\prime}}
$$


and hence the estimate asserted follows without assuming quasi-uniformity.

Since $\int_{\square_{P}^{\prime}}(d(P)-d) u_{x} d V$ vanishes for $d=1$, we find with the Bramble - Hilbert lemma

$$
\left|F_{2}\right| \leqslant C\left(\sum_{P \in \Omega_{1 / 2}^{x}}\left|\square_{P}^{\prime}\right|^{-1+s} \max \left(h_{P},\left|S_{P}^{\prime}\right|\right)^{2}\left\|u_{x}\right\|_{s, 2, \square_{P}^{\prime}}^{2}\right)^{1 / 2}\left\|P_{H} w_{H}\right\|_{0,2}
$$

which gives the estimate wanted, again without assuming quasi-uniformity if $s=1$.

Regarding $F_{3}$, we firstly observe that

$$
F_{3}=\frac{1}{2} \sum_{P \in \Omega_{1 / 2}^{x}}\left(\int_{\square_{P}^{l}} d u_{x} d V-\int_{\square_{P}^{r}} d u_{x} d V\right) h_{P} \delta_{x}^{(1 / 2)} \bar{w}_{P}
$$

where $\square_{P}^{r}$ and $\square_{P}^{l}$ denotes the part of $\square_{P}^{\prime}$ to the right and left of the vertical line through $P \in \Omega_{1 / 2}^{x}$, respectively. So, we come up with

$$
\left|F_{3}\right| \leqslant \frac{1}{2} \sum_{P \in \Omega_{1 / 2}^{x}} h_{P} \int_{\square_{P}^{\prime}}\left|d u_{x}\right| d V\left|\delta_{x}^{(1 / 2)} w_{P}\right| \leqslant C\left(\sum_{P \in \Omega_{1 / 2}^{x}} h_{P}^{2} \int_{\square_{P}^{\prime}}\left|d u_{x}\right|^{2} d V \sum_{P \in \Omega_{1 / 2}^{x}}\left|\square_{P}^{\prime}\right|\left|\delta_{x}^{(1 / 2)} w_{P}\right|^{2}\right)^{1 / 2}
$$

which gives, because of $d \in \mathcal{C}(\bar{\Omega})$ and $\|\cdot\|_{0,2, \square_{P}} \leqslant\|\cdot\|_{s, 2, \square_{P}}$, with (4.9)

$$
\left|F_{3}\right| \leqslant C\left(\sum_{P \in \Omega_{1 / 2}^{x}} h_{P}^{2}\left\|u_{x}\right\|_{s, 2, \square_{P}}^{2}\right)^{1 / 2}\left|P_{H} w_{H}\right|_{1,2}
$$

With respect to the term $G$, we remember that $\left(P_{H} \bar{w}_{H}\right)\left(P^{V}\right)=\left(\bar{w}_{P I I}+\bar{w}_{P I I I}\right) / 2$ and $\left|\triangle_{P}\right|=4\left|\triangle_{P}^{I}\right|=4\left|\triangle_{P}^{I I}\right|=2\left|\square_{P}^{I I I}\right|$ for $P \in \Gamma_{1 / 2}^{x y}$. We thus find $G=\sum_{P \in \Gamma_{1 / 2}^{x y}}\left(G_{P, 1}+G_{P, 2}\right)$ with

$$
\begin{gathered}
G_{P, 1}:=\left(\left|\triangle_{P}^{I}\right| d\left(P^{V}\right) \delta_{x}^{(1 / 2)} u_{P^{V}}-\int_{\triangle_{P}^{I}} d u_{x} d V\right) \bar{w}_{P^{I}}+ \\
+\left(\left|\triangle_{P}^{I I}\right| d\left(P^{V}\right) \delta_{x}^{(1 / 2)} u_{P^{V}}-\int_{\triangle_{P}^{I I}} d u_{x} d V\right) \bar{w}_{P^{I I}}+\left(\left|\square_{P}^{I I I}\right| d\left(P^{V}\right) \delta_{x}^{(1 / 2)} u_{P^{V}}-\int_{\square_{P}^{I I I}} d u_{x} d V\right) \bar{w}_{P^{I I I}}, \\
G_{P, 2}:=\frac{\left|\triangle_{P}\right|}{4} d\left(P^{V}\right) \delta_{x}^{(1 / 2)} u_{P^{V}}\left(\bar{w}_{P^{I I}}-\bar{w}_{P^{I}}\right) .
\end{gathered}
$$

With the same arguments as used in the first part of this proof, we find an optimal estimate for $G_{P, 1}$. Since $P_{H} w_{H} \in H^{1}(\Omega) \hookrightarrow H^{1 / 2}(\Gamma)$ with

$$
\left|P_{H} w_{H}\right|_{1 / 2,2, \Gamma_{P}}=\left|w_{P^{I}}-w_{P^{I I}}\right| \text { for } P \in \Gamma_{1 / 2},
$$

it can be shown (with an argument analogous to (4.8)) that

$$
\left|G_{P, 2}\right| \leqslant C\left|\triangle_{P}\right|^{1 / 2}\left\|u_{x}\right\|_{s, 2, \triangle_{P}}\left|P_{H} w_{H}\right|_{1 / 2,2, \Gamma_{P}} .
$$

The assertion finally follows from the application of the Cauchy-Schwarz inequality.

The following result is already known from [11, Lemma 4.5] for a domain that is the union of rectangles. In the case of oblique boundary parts, the proof follows the same lines and shall be omitted. 
Lemma 4.4. Let $s \in(1 / 2,1], u \in H^{1+s}(\Omega), f \in W^{1,2 /(1-s)}(\Omega)$, and assume that $\left\{\bar{\Omega}_{H}\right\}_{H \in \Lambda}$ is quasi-uniform if $s \neq 1$. Then for all $w_{H} \in W_{H}$

$$
\left|\tau_{H}^{(f)}\left(w_{H}\right)\right| \leqslant C\left(\sum_{P \in \bar{\Omega}_{H}}\left(\operatorname{diam} \square_{P}\right)^{2 s}\|u\|_{1+s, 2, \square_{P}}^{2}\right)^{1 / 2}\left\|P_{H} w_{H}\right\|_{0,2} .
$$

For the boundary terms, we finally have the following result.

Lemma 4.5. Let $s \in(1 / 2,1], u \in H^{1+s}(\Omega), \alpha \in W^{1,1 /(1-s)}(\Gamma), \psi \in H^{s}(\Gamma)$, and assume that $\left\{\bar{\Omega}_{H}\right\}_{H \in \Lambda}$ is quasi-uniform if $s \neq 1$. Then for all $w_{H} \in W_{H}$

$$
\left|\tau_{\Gamma, H}\left(w_{H}\right)\right| \leqslant\left(\sum_{P \in \Gamma_{H}}\left|\Gamma_{P}\right|^{2 s}\left(\|u\|_{s, 2, \Gamma_{P}}^{2}+\|\psi\|_{s, 2, \Gamma_{P}}^{2}\right)\right)^{1 / 2}\left\|P_{H} w_{H}\right\|_{0,2, \Gamma} .
$$

We omit the proof here as it follows the same arguments as [11, Lemmata 4.6, 4.7].

Proof of Theorem 2.1. Theorem 2.1 immediately follows from the lemmata in this section together with Proposition 2.1 upon noting that for the terms with $c$ and $e$ analogous results as for $a$ and $d$, respectively, are at hand. Remember here that $\|\cdot\|_{s, 2, \Gamma}$ is the Euclidean sum of the corresponding norm taken over all straight boundary sections of $\Gamma$.

\section{High-order convergence: proof of Theorem 2.2}

Since $\tau_{\Gamma, H}$ from (4.5) only admits a first-order estimate, we have to rely upon another decomposition of the truncation error. The main idea is to correct $\tau_{\Gamma, H}$ so that it becomes of higher order and then to allocate this correction to the the truncation error related to the main part of the differential operator.

A simple calculation shows that for all $w_{H} \in W_{H}$ and an appropriate function $\phi$

$$
\begin{gathered}
\sum_{P \in \Gamma_{H}}\left(\int_{\Gamma_{P}} \phi d \sigma-\left|\Gamma_{P}\right| \phi(P)\right) \bar{w}_{P}=\sum_{P \in \Gamma_{1 / 2}}\left(\int_{\Gamma_{P}} \phi d \sigma-\frac{\left|\Gamma_{P}\right|}{2}\left(\phi\left(P^{I}\right)+\phi\left(P^{I I}\right)\right)\right) \frac{\bar{w}_{P^{I}}+\bar{w}_{P^{I I}}}{2}+ \\
+\sum_{P \in \Gamma_{1 / 2}}\left(\int_{\Gamma_{P}^{I}} \phi d \sigma-\frac{\left|\Gamma_{P}\right|}{2} \phi\left(P^{I}\right)-\int_{\Gamma_{P}^{I I}} \phi d \sigma+\frac{\left|\Gamma_{P}\right|}{2} \phi\left(P^{I I}\right)\right) \frac{\bar{w}_{P^{I}}-\bar{w}_{P^{I I}}}{2}
\end{gathered}
$$

In the following, let for $w_{H} \in W_{H}$ and an appropriate function $\phi$

$$
S_{\Gamma, H}^{x}\left(\phi, w_{H}\right):=\sum_{P \in \Gamma_{1 / 2}} \eta_{x}(P)\left(\int_{\Gamma_{P}^{I}} \phi d \sigma-\frac{\left|\Gamma_{P}\right|}{2} \phi\left(P^{I}\right)-\int_{\Gamma_{P}^{I I}} \phi d \sigma+\frac{\left|\Gamma_{P}\right|}{2} \phi\left(P^{I I}\right)\right) \frac{\bar{w}_{P I}-\bar{w}_{P I I}}{2} .
$$

With

$$
\begin{gathered}
\tilde{\tau}_{\Gamma, H}\left(w_{H}\right):=\sum_{P \in \Gamma_{1 / 2}}\left(\int_{\Gamma_{P}}(\psi-\alpha u) d \sigma-\frac{\left|\Gamma_{P}\right|}{2}\left((\psi-\alpha u)\left(P^{I}\right)+(\psi-\alpha u)\left(P^{I I}\right)\right)\right) \frac{\bar{w}_{P I}+\bar{w}_{P I I}}{2}, \\
\tilde{\tau}_{H}^{(a)}\left(w_{H}\right):=\tau_{H}^{(a)}\left(w_{H}\right)+S_{\Gamma, H}^{x}\left(a u_{x}, w_{H}\right),
\end{gathered}
$$

and analogous definitions for $\tilde{\tau}_{H}^{(b)}$ (see also (5.11) below) and $\tilde{\tau}_{H}^{(c)}$, we find from (1.2) and $(5.1)$

$$
\tau_{H}\left(w_{H}\right)=\tilde{\tau}_{H}^{(a)}\left(w_{H}\right)+\tilde{\tau}_{H}^{(b)}\left(w_{H}\right)+\tilde{\tau}_{H}^{(c)}\left(w_{H}\right)+\tau_{H}^{(d)}\left(w_{H}\right)+\tau_{H}^{(e)}\left(w_{H}\right)+\tau_{H}^{(f)}\left(w_{H}\right)+\tilde{\tau}_{\Gamma, H}\left(w_{H}\right)
$$

in place of (4.1). Since $\tilde{\tau}_{\Gamma, H}$ consists of one-dimensional trapezoidal rules, we find 
Lemma 5.1. Let $s \in(1,2], u \in H^{1+s}(\Omega), \alpha \in W^{2,1 /(2-s)}(\Gamma), \psi \in H^{s}(\Gamma)$. Then for all $w_{H} \in W_{H}$

$$
\left|\tilde{\tau}_{\Gamma, H}\left(w_{H}\right)\right| \leqslant C\left(\sum_{P \in \Gamma_{1 / 2}}\left|\Gamma_{P}\right|^{2 s}\left(\|u\|_{s, 2, \Gamma_{P}}^{2}+\|\psi\|_{s, 2, \Gamma_{P}}^{2}\right)\right)^{1 / 2}\left\|P_{H} w_{H}\right\|_{1,2} .
$$

The proof, which is omitted here, relies upon the estimate

$$
\left|\Gamma_{P}\right|\left(\left|w_{P^{I}}\right|^{2}+\left|w_{P I I}\right|^{2}\right) \leqslant C\left\|P_{H} w_{H}\right\|_{0,2, \Gamma_{P}}^{2} \leqslant C\left\|P_{H} w_{H}\right\|_{1,2}^{2}
$$

and follows the same arguments as the proof of [11, Lemma 5.8]. Further, the continuous embedding $H^{1+s}(\Omega) \hookrightarrow H^{s}\left(\Gamma_{P}\right)$ is employed. Note that the assumption $\psi \in H^{s}(\Gamma)$ is essential and does not follow from the regularity of the solution and coefficients together with (1.2).

In what follows, we often make use of an estimate of the $L^{2}$-norm on a boundary strip.

Lemma 5.2. For $v \in H^{t}(\Omega)(0 \leqslant t \leqslant 1)$, the following estimate holds true:

$$
\left(\sum_{P \in \Gamma_{1 / 2}^{x y}}\|v\|_{0,2, \triangle_{P}}^{2}\right)^{1 / 2} \leqslant c\left(H_{\max }\right)\|v\|_{t, 2, \Omega} \quad \text { with } \quad c(h)=C \begin{cases}h^{\min (t, 1 / 2)} & \text { if } t \neq 1 / 2 \\ h^{1 / 2}|\log h| & \text { if } t=1 / 2 .\end{cases}
$$

Proof. Since the polygonal domain $\Omega$ possesses only a fixed number of oblique boundary sections, it suffices to consider one of them. So, let $\Gamma^{\prime} \subset \Gamma$ be one of the oblique boundary sections. Then all triangles $\triangle \in \mathcal{T}_{H}^{\text {obl }}$ having an oblique edge on $\Gamma^{\prime}$ lie within a trapezoidal domain $\Omega^{\prime}, H_{\max } \subset \Omega$ with $\operatorname{dist}\left(x, \Gamma^{\prime}\right)<H_{\max }$ for $x \in \Omega^{\prime}, H_{\max }$. We can construct a new domain $\Omega^{\prime \prime}$ of class $\mathcal{C}^{1}$ such that $\Gamma^{\prime} \subset \partial \Omega^{\prime \prime}$ and $\Omega^{\prime, H_{\max }} \subset \Omega^{\prime \prime}$ (obviously, $\Omega^{\prime \prime}$ is not contained in $\Omega$ ). Since $\Omega^{\prime}, H_{\max }$ is contained within a strip $\Omega^{\prime \prime}, H_{\max } \subset \Omega^{\prime \prime}$ of width $H_{\max }$ along $\partial \Omega^{\prime \prime}$, Il'in's result [20, Thm. 4.4 and Rem. 12.4] for domains of class $\mathcal{C}^{1}$ gives

$\sum_{P \in \Gamma_{1 / 2}^{x y} \cap \Gamma^{\prime}}\|v\|_{0,2, \triangle_{P}}^{2} \leqslant\|v\|_{0,2, \Omega^{\prime}, H_{\max }}^{2} \leqslant\|v\|_{0,2, \Omega^{\prime \prime}, H_{\max }}^{2} \leqslant c\left(H_{\max }\right)^{2}\|v\|_{t, 2, \Omega^{\prime \prime}}^{2} \leqslant C c\left(H_{\max }\right)^{2}\|v\|_{t, 2, \Omega}^{2}$.

In the last step, we have employed the fact that since $\Omega$ is of class $\mathrm{e}^{0+1}$, we can extend $v$ to a function on $\mathbb{R}^{2}$ such that $\|v\|_{t, 2, \mathbb{R}^{2}} \leqslant C\|v\|_{t, 2, \Omega}$ (cf. [42, Thm. A.4]).

For $t \in[0,1 / 2)$, the estimate above also follows from the continuous embedding of $H^{t}(\Omega)$ into the Lebesgue space $L_{\rho}^{2}(\Omega)$ with the weight function $\rho:=\operatorname{dist}(\cdot, \Gamma)^{-2 t}$ (cf. [42, Lemma 3.32]).

From [11, Sect. 5], we already have an optimal estimate of order $\mathcal{O}\left(H_{\max }^{s}\right)$ for $\tilde{\tau}_{H}^{(a)}$ but only on a domain that is the union of rectangles. Unfortunately, in the case of oblique boundary parts, we can only prove a suboptimal estimate. Remember in the following that $\Gamma_{1 / 2}^{x y}=\varnothing$ if oblique boundary parts do not occur.

Lemma 5.3. Let $s \in(1,2], u \in H^{1+s}(\Omega), a \in W^{2,2 /(2-s)}(\Omega)$, and assume that $\left\{\bar{\Omega}_{H}\right\}_{H \in \Lambda}$ is quasi-uniform if $s \neq 2$. Then for all $w_{H} \in W_{H}$

$$
\begin{aligned}
\left|\tilde{\tau}_{H}^{(a)}\left(w_{H}\right)\right| & \leqslant C\left(\sum_{P \in \Omega_{1 / 2}^{y}}\left(\operatorname{diam} \square_{P}\right)^{2 s}\left\|u_{x}\right\|_{s, 2, \square_{P}}^{2}+\sum_{P \in \Gamma_{1 / 2}^{x y}}\left(\left|\Gamma_{P}\right|^{2 s}\left\|u_{x}\right\|_{s, 2, \triangle_{P}}^{2}+\left|\Gamma_{P}\right|^{2}\left\|u_{x}\right\|_{1,2, \triangle_{P}}^{2}\right)\right)^{1 / 2} \times \\
& \times\left\|P_{H} w_{H}\right\|_{1,2} \leqslant C\|u\|_{1+s, 2}\left\|P_{H} w_{H}\right\|_{1,2} \begin{cases}H_{\max }^{\min (s, 3 / 2)} & \text { if } s \neq 3 / 2, \\
H_{\max }^{3 / 2}\left|\log H_{\max }\right| & \text { if } s=3 / 2 .\end{cases}
\end{aligned}
$$

If $\Omega$ is the union of rectangles (i.e., $\left.\Gamma_{1 / 2}^{x y}=\varnothing\right)$ the local estimate above yields order $\mathcal{O}\left(H_{\max }^{s}\right)$. 
Proof. From (4.2), we find

$$
\tau_{H}^{(a)}\left(w_{H}\right)=\sum_{P \in \Omega_{1 / 2}^{x}} h_{P}\left(\left|S_{P}\right| a(P) \delta_{x}^{(1 / 2)} u_{P}-\int_{S_{P}} a u_{x} d y\right) \delta_{x}^{(1 / 2)} \bar{w}_{P}
$$

The generalised Bramble - Hilbert lemma yields for $P=\left(x_{j+1 / 2}, y_{\ell}\right) \in \Omega_{1 / 2}^{x}$ (which is in general not the midpoint of $S_{P}$ )

$$
\begin{gathered}
\left(h_{P}\left|S_{P}\right|\right)^{1 / 2}\left|\delta_{x}^{(1 / 2)} u_{P}-u_{x}(P)\right|=\left(h_{P}\left|S_{P}\right|\right)^{1 / 2}\left|\frac{1}{h_{j}} \int_{x_{j}}^{x_{j+1}}\left(u_{x}\left(x, y_{\ell}\right)-u_{x}\left(x_{j+1 / 2}, y_{\ell}\right)\right) d x\right| \leqslant \\
\leqslant C \max \left(h_{P},\left|S_{P}\right|\right)^{s}\left|u_{x}\right|_{s, 2, \square_{P}},
\end{gathered}
$$

where quasi-uniformity is not needed if $s=2$. Note that the result remains true if one replaces $\square_{P}$ by its lower or upper half. Hence, we can replace $\delta_{x}^{(1 / 2)} u_{P}$ in (5.4) by $u_{x}(P)$ committing an error of the correct order. With

$$
h_{j}\left(\delta_{x}^{(1 / 2)} \bar{w}_{j+1 / 2, \ell+1}-\delta_{x}^{(1 / 2)} \bar{w}_{j+1 / 2, \ell}\right)=k_{\ell}\left(\delta_{y}^{(1 / 2)} \bar{w}_{j+1, \ell+1 / 2}-\delta_{y}^{(1 / 2)} \bar{w}_{j, \ell+1 / 2}\right)
$$

and summation by parts, we find

$$
\sum_{P \in \Omega_{1 / 2}^{x}} h_{P}\left(\left|S_{P}\right|\left(a u_{x}\right)(P)-\int_{S_{P}} a u_{x} d y\right) \delta_{x}^{(1 / 2)} \bar{w}_{P}=F_{1}+F_{2}+F_{3}+F_{4}+F_{5},
$$

where

$$
\begin{gathered}
F_{1}:=\sum_{P \in \Omega_{1 / 2}^{x y} \backslash \Gamma_{1 / 2}^{x y}} h_{P}\left(\frac{k_{P}}{2}\left(\left(a u_{x}\right)\left(P^{+}\right)+\left(a u_{x}\right)\left(P^{-}\right)\right)-\int_{S_{P}} a u_{x} d y\right) \frac{\delta_{x}^{(1 / 2)} \bar{w}_{P^{+}}+\delta_{x}^{(1 / 2)} \bar{w}_{P^{-}}}{2}, \\
F_{2}:=\sum_{P \in \Omega_{1 / 2}^{y}} \sum_{i=1}^{4}(-1)^{i} k_{P}\left(\frac{k_{P}}{2}\left(a u_{x}\right)\left(P^{i}\right)-\int_{S_{P}^{i}} a u_{x} d y\right) \frac{\delta_{y}^{(1 / 2)} \bar{w}_{P}}{2}, \\
F_{3}:=\sum_{P \in \Gamma_{1 / 2}^{x y}} \operatorname{sgn} \eta_{x}(P)\left(\frac{k_{P}}{2}\left(a u_{x}\right)\left(P^{I}\right)-\int_{S_{P}^{I}} a u_{x} d y-\frac{k_{P}}{2}\left(a u_{x}\right)\left(P^{I I I}\right)+\int_{S_{P}^{I I I}} a u_{x} d y\right) \frac{\bar{w}_{P^{I}}-\bar{w}_{P}{ }^{I I I}}{2}, \\
F_{5}:=\sum_{P \in \Gamma_{1 / 2}^{y}} \eta_{x}(P)\left(\frac{k_{P}}{2}\left(a u_{x}\right)\left(P^{I}\right)-\int_{S_{P}^{I I}} a u_{x} d y-\frac{k_{P}}{2}\left(a u_{x}\right)\left(P^{I I}\right)+\int_{\Gamma_{P}^{I I}} a u_{x} d y\right)\left(P_{P^{I I}}-\bar{w}_{P^{I}}-\bar{w}_{P^{I I I}}\right)
\end{gathered}
$$

Since $\Omega_{1 / 2}^{y}$ and $\Omega_{1 / 2}^{x y} \backslash \Gamma_{1 / 2}^{x y}$ describe the union of rectangles, we can apply [11, Lemmata 5.3, 5.4] in order to optimally estimate $F_{1}$ and $F_{2}$ (if $s=2$ without quasi-uniformity). Altogether, we find

$$
\left|\tilde{\tau}_{H}^{(a)}\left(w_{H}\right)\right| \leqslant C\left(\sum_{P \in \Omega_{1 / 2}^{y}}\left(\operatorname{diam} \square_{P}\right)^{2 s}\left\|u_{x}\right\|_{s, 2, \square_{P}}^{2}\right)^{1 / 2}\left|P_{H} w_{H}\right|_{1,2}+\left|F_{3}+F_{4}+F_{5}+S_{\Gamma, H}^{x}\left(a u_{x}, w_{H}\right)\right| .
$$


Since $\eta_{x}(P)=\operatorname{sgn} \eta_{x}(P) k_{P} /\left|\Gamma_{P}\right|\left(P \in \Gamma_{1 / 2}^{x y}\right)$, we have

$$
F_{3}+F_{4}+F_{5}+S_{\Gamma, H}^{x}\left(a u_{x}, w_{H}\right)=\frac{1}{2} \sum_{P \in \Gamma_{1 / 2}^{x y}} \operatorname{sgn} \eta_{x}(P)\left(G_{P, 1}+G_{P, 2}\right)
$$

with

$$
\begin{gathered}
G_{P, 1}:=\left(\frac{k_{P}}{4}\left(a u_{x}\right)\left(P^{I}\right)+\frac{k_{P}}{4}\left(a u_{x}\right)\left(P^{I V}\right)-\int_{S_{P}^{I}} a u_{x} d y-\frac{k_{P}}{4}\left(a u_{x}\right)\left(P^{I I I}\right)-\frac{k_{P}}{4}\left(a u_{x}\right)\left(P^{I V}\right)+\int_{S_{P}^{I I I}} a u_{x} d y\right)+ \\
+2\left(\frac{k_{P}}{4}\left(a u_{x}\right)(P)+\frac{k_{P}}{4}\left(a u_{x}\right)\left(P^{V}\right)-\int_{S_{P}^{I I}} a u_{x} d y\right)\left(\bar{w}_{P^{I I}}-\bar{w}_{P} I I I\right)+\left(\frac{k_{P}}{\left|\Gamma_{P}\right|} \int_{\Gamma_{P}^{I}} a u_{x} d \sigma-\frac{k_{P}}{4}\left(a u_{x}\right)\left(P^{I}\right)-\right. \\
\left.-\frac{k_{P}}{4}\left(a u_{x}\right)(P)-\frac{k_{P}}{\left|\Gamma_{P}\right|} \int_{\Gamma_{P}^{I I}} a u_{x} d \sigma+\frac{k_{P}}{4}\left(a u_{x}\right)\left(P^{I I}\right)+\frac{k_{P}}{4}\left(a u_{x}\right)(P)\right)\left(\bar{w}_{P^{I}}-\bar{w}_{P^{I I}}\right), \\
G_{P, 2}:=\frac{k_{P}}{4}\left(\left(a u_{x}\right)\left(P^{I}\right)-\left(a u_{x}\right)\left(P^{I I I}\right)\right)\left(\bar{w}_{P^{I}}-\bar{w}_{P}^{I I I}\right)+\frac{k_{P}}{2}\left(\left(a u_{x}\right)\left(P^{V}\right)-\left(a u_{x}\right)(P)\right)\left(\bar{w}_{P^{I I}}-\bar{w}_{P}^{I I I}\right)+ \\
+\frac{k_{P}}{4}\left(\left(a u_{x}\right)\left(P^{I I}\right)-\left(a u_{x}\right)\left(P^{I}\right)\right)\left(\bar{w}_{P^{I}}-\bar{w}_{P^{I I}}\right) .
\end{gathered}
$$

Obviously, $G_{P, 1}$ consists of one-dimensional trapezoidal rules. Arguing similarly as in [11, Lemma 5.3], we find (with $\operatorname{diam} \triangle_{P}=\left|\Gamma_{P}\right|$ for $P \in \Gamma_{1 / 2}^{x y}$ ) the optimal estimate

$$
\left|G_{P, 1}\right| \leqslant C k_{P}^{1 / 2} h_{P}^{-1 / 2}\left|\Gamma_{P}\right|^{s}\left\|u_{x}\right\|_{s, 2, \triangle_{P}}\left(\left|P_{H} w_{H}\right|_{1,2, \triangle_{P}}+\left|P_{H} w_{H}\right|_{1 / 2,2, \Gamma_{P}}\right) .
$$

Note that quasi-uniformity is not needed if $s=2$. The estimate of the one-dimensional trapezoidal rules in the $y$-direction leads indeed to a factor $\left(k_{P} / h_{P}\right)^{1 / 2}$ that cannot be absorbed if it appears in connection with $\bar{w}_{P^{I}}-\bar{w}_{P^{I I I}}$ since

$$
\begin{gathered}
\left|w_{P^{I}}-w_{P I I I}\right| \leqslant\left|w_{P^{I}}-w_{P I I}\right|+\left|w_{P I I}-w_{P I I I}\right| \leqslant \\
\leqslant C\left(\left|P_{H} w_{H}\right|_{1 / 2,2, \Gamma_{P}}+h_{P}^{1 / 2} k_{P}^{-1 / 2}\left|P_{H} w_{H}\right|_{1,2, \triangle_{P}}\right)
\end{gathered}
$$

is the best possible estimate (remember here (4.13)). But since $P \in \Gamma_{1 / 2}^{x y}$, the quotient $k_{P} / h_{P}$ is just a constant slope of the corresponding oblique boundary section of $\Gamma$.

Regarding $G_{P, 2}$, one can show that

$$
\left|2\left(\left(a u_{x}\right)\left(P^{V}\right)-\left(a u_{x}\right)(P)\right)+\left(a u_{x}\right)\left(P^{I}\right)-\left(a u_{x}\right)\left(P^{I I I}\right)\right| \leqslant C\left|\triangle_{P}\right|^{-1 / 2}\left|\Gamma_{P}\right|^{s}\left\|u_{x}\right\|_{s, 2, \triangle_{P}} .
$$

Here, for $1<s<2$, we use $u_{x} \in H^{s}(\Omega) \hookrightarrow \mathcal{C}(\bar{\Omega})$ and a Taylor expansion of $a \in$ $W^{2,2 /(2-s)}(\Omega) \hookrightarrow \mathcal{C}^{1}(\bar{\Omega})$. Upon noting $\left\|a u_{x}\right\|_{2,2, \triangle_{P}} \leqslant C\|a\|_{2, \infty}\left\|u_{x}\right\|_{2,2, \triangle_{P}}$, the estimate again holds without assuming quasi-uniformity if $s=2$. So, we can replace $2\left(\left(a u_{x}\right)\left(P^{V}\right)-\left(a u_{x}\right)(P)\right)$ by $-\left(\left(a u_{x}\right)\left(P^{I}\right)-\left(a u_{x}\right)\left(P^{I I I}\right)\right)$ at the expense of an error having the correct order.

It thus remains to estimate

$$
G_{P, 2}^{\prime}:=\frac{k_{P}}{4}\left(\left(a u_{x}\right)\left(P^{I I}\right)-\left(a u_{x}\right)\left(P^{I I I}\right)\right)\left(\bar{w}_{P^{I}}-\bar{w}_{P I I}\right) .
$$


The Bramble - Hilbert lemma and (4.13) only allow to prove the suboptimal estimate

$$
\left|G_{P, 2}^{\prime}\right| \leqslant C k_{P}^{1 / 2} h_{P}^{-1 / 2}\left|\Gamma_{P}\right|\left\|u_{x}\right\|_{1,2, \triangle_{P}}\left|P_{H} w_{H}\right|_{1 / 2,2, \Gamma_{P}} .
$$

Here, we used the fact that $a \in W^{2,2 /(2-s)}(\Omega) \hookrightarrow \mathcal{C}^{1}(\bar{\Omega})$ and $u_{x} \in H^{s}(\Omega) \hookrightarrow H^{1}(\Omega)$ implies $\left|a u_{x}\right|_{1,2, \triangle_{P}} \leqslant C\|a\|_{1, \infty}\left\|u_{x}\right\|_{1,2, \triangle_{P}}$. Note that estimate (5.10) again does not require quasiuniformity if $s=2$ since $k_{P} / h_{P}$ is the slope of the corresponding oblique boundary section of $\Gamma$.

The global estimate asserted can be derived from the preceding estimates and Lemma 5.2.

For the truncation error due to mixed derivatives, we have the following result.

Lemma 5.4. Let $s \in(1,2], u \in H^{1+s}(\Omega), b \in W^{2,2 /(2-s)}(\Omega)$, and assume that $\left\{\bar{\Omega}_{H}\right\}_{H \in \Lambda}$ is quasi-uniform if $s \neq 2$. Then for all $w_{H} \in W_{H}$

$$
\begin{gathered}
\left|\tilde{\tau}_{H}^{(b)}\left(w_{H}\right)\right| \leqslant C\left(\sum_{P \in \bar{\Omega}_{H}}\left(\operatorname{diam} \square_{P}\right)^{2 s}\|u\|_{1+s, 2, \square_{P}}^{2}+\sum_{P \in \Gamma_{1 / 2}^{x} \cup \Gamma_{1 / 2}^{y}}\left|\Gamma_{P}\right|^{\min (2 s, 3)}\|u\|_{1 / 2+s, 2, \Gamma_{P}}^{2}+\right. \\
\left.+\sum_{P \in \Gamma_{1 / 2}^{x y}}\left(\left|\Gamma_{P}\right|^{2 s}\|u\|_{1+s, 2, \triangle_{P}}^{2}+\left|\Gamma_{P}\right|^{2}\|u\|_{2,2, \triangle_{P}}^{2}+\left|\Gamma_{P}\right|^{3}\|u\|_{1 / 2+s, 2, \Gamma_{P}}^{2}\right)\right)^{1 / 2}\left\|P_{H} w_{H}\right\|_{1,2} \leqslant \\
\leqslant C\|u\|_{1+s, 2}\left\|P_{H} w_{H}\right\|_{1,2} \begin{cases}H_{\max }^{\min (s, 3 / 2)} & \text { if } s \neq 3 / 2, \\
H_{\max }^{3 / 2}\left|\log H_{\max }\right| & \text { if } s=3 / 2 .\end{cases}
\end{gathered}
$$

If $\Omega$ is the union of rectangles (i.e., $\Gamma_{1 / 2}^{x y}=\varnothing$ ), then the local estimate above yields order $\mathcal{O}\left(H_{\max }^{\min (s, 3 / 2)}\right)$.

Proof. As in the proof of Lemma 4.2, we concentrate on the contributions due to $-\left(b u_{y}\right)_{x}$ that are denoted by a superscript $y x$. We have for all $w_{H} \in W_{H}$

$$
\tilde{\tau}_{H}^{(b, y x)}\left(w_{H}\right)=\tau_{H}^{(b, y x)}\left(w_{H}\right)+S_{\Gamma, H}^{x}\left(b u_{y}, w_{H}\right),
$$

where $\tau_{H}^{(b, y x)}\left(w_{H}\right)$ is given by (4.6) and (4.7). Let $F:=\tau_{H}^{(b, y x)}\left(w_{H}\right)-G_{1}$ with $G_{1}$ given by (4.10). We then find from (4.6) and (4.7)

$$
\begin{aligned}
& F=\sum_{P \in \Omega_{1 / 2}^{x y} \backslash \Gamma_{1 / 2}^{x y}} \frac{\left|\square_{P}\right|}{4}\left(b\left(P^{1}\right) \delta_{y}^{(1 / 2)} u_{P^{r}}+b\left(P^{2}\right) \delta_{y}^{(1 / 2)} u_{P^{l}}+b\left(P^{3}\right) \delta_{y}^{(1 / 2)} u_{P^{l}}+b\left(P^{4}\right) \delta_{y}^{(1 / 2)} u_{P^{r}}-\frac{4}{k_{P}} \int_{S_{P}} b u_{y} d y\right) \times \\
& \quad \times \frac{\delta_{x}^{(1 / 2)} \bar{w}_{P^{+}}+\delta_{x}^{(1 / 2)} \bar{w}_{P^{-}}}{2}+\sum_{P \in \Omega_{1 / 2}^{x y} \backslash \Gamma_{1 / 2}^{x y}} \frac{\left|\square_{P}\right|}{4}\left(b\left(P^{1}\right) \delta_{y}^{(1 / 2)} u_{P^{r}}+b\left(P^{2}\right) \delta_{y}^{(1 / 2)} u_{P^{l}}-\frac{4}{k_{P}} \int_{S_{P}^{+}} b u_{y} d y-\right. \\
& \left.\quad-b\left(P^{3}\right) \delta_{y}^{(1 / 2)} u_{P^{l}}-b\left(P^{4}\right) \delta_{y}^{(1 / 2)} u_{P^{r}}+\frac{4}{k_{P}} \int_{S_{P}^{-}} b u_{y} d y\right) \frac{\delta_{x}^{(1 / 2)} \bar{w}_{P^{+}}-\delta_{x}^{(1 / 2)} \bar{w}_{P^{-}}}{2}=: F_{1}+F_{2} . \quad
\end{aligned}
$$

For the sum $F_{1}$ in (5.12), we firstly conclude from the Bramble - Hilbert lemma and (4.8) $\left|\square_{P}\right|^{1 / 2}\left|b\left(P^{1}\right)+b\left(P^{4}\right)-2 b\left(P^{r}\right)\right|\left|\delta_{y}^{(1 / 2)} u_{P^{r}}\right| \leqslant C\left|\square_{P}\right|^{-(2-s) / 2} \max \left(h_{P}, k_{P}\right)^{2}|b|_{2,2 /(2-s)}\left\|u_{y}\right\|_{s, 2, \square_{P}}$ since $b \in W^{2,2 /(2-s)}(\Omega) \hookrightarrow \mathcal{C}(\bar{\Omega})$ and $u_{y} \in H^{s}(\Omega)$. The application of the Cauchy - Schwarz inequality and (4.9) thus shows that the error that appears when replacing $b\left(P^{1}\right)+b\left(P^{4}\right)$ 
by $2 b\left(P^{r}\right)$ and, analogously, $b\left(P^{2}\right)+b\left(P^{3}\right)$ by $2 b\left(P^{l}\right)$ in $F_{1}$ can be bounded as desired. Here, quasi-uniformity of $\left\{\bar{\Omega}_{H}\right\}_{H \in \Lambda}$ is only needed if $s \neq 2$. For $P=\left(x_{j+1 / 2}, y_{\ell+1 / 2}\right) \in \Omega_{1 / 2}^{x y}$, the quantity

$$
\begin{gathered}
\left(b \delta_{y}^{(1 / 2)} u_{H}\right)_{P^{r l}}-b_{P^{r l}}\left(\delta_{y}^{(1 / 2)} u_{H}\right)_{P^{r l}}=\frac{1}{2 k_{P}} \int_{y_{\ell}}^{y_{\ell+1}}\left(b\left(x_{j+1}, y_{\ell+1 / 2}\right) u_{y}\left(x_{j+1}, y\right)+\right. \\
\left.+b\left(x_{j}, y_{\ell+1 / 2}\right) u_{y}\left(x_{j}, y\right)-\frac{1}{2}\left(b\left(x_{j+1}, y_{\ell+1 / 2}\right)+b\left(x_{j}, y_{\ell+1 / 2}\right)\right)\left(u_{y}\left(x_{j+1}, y\right)+u_{y}\left(x_{j}, y\right)\right)\right) d y
\end{gathered}
$$

vanishes for $b=1$ and arbitrary $u_{y}$ as well as for arbitrary $b$ and $u_{y}=1$, and is bounded for $b \in W^{2,2 /(2-s)}(\Omega) \hookrightarrow \mathrm{C}^{1}(\bar{\Omega}), u_{y} \in H^{s}(\Omega) \hookrightarrow H^{1}(\Omega)$. The bilinear lemma yields

$$
\begin{gathered}
\left|\square_{P}\right|^{1 / 2}\left|\left(b \delta_{y}^{(1 / 2)} u_{H}\right)_{P^{r l}}-b_{P^{r l}}\left(\delta_{y}^{(1 / 2)} u_{H}\right)_{P^{r l}}\right| \leqslant C \max \left(h_{P}, k_{P}\right)^{2}|b|_{1, \infty}\left|u_{y}\right|_{1,2, \square_{P}} \\
\leqslant C \max \left(h_{P}, k_{P}\right)^{2}|b|_{1, \infty}\left(\left\|u_{y x}\right\|_{s-1,2, \square_{P}}^{2}+\left\|u_{y y}\right\|_{s-1,2, \square_{P}}^{2}\right)^{1 / 2} .
\end{gathered}
$$

We further have with (4.8)

$$
\left|\square_{P}\right|^{1 / 2}\left|b_{P^{r l}}-b(P)\right|\left|\left(\delta_{y}^{(1 / 2)} u_{H}\right)_{P^{r l}}\right| \leqslant C\left|\square_{P}\right|^{-(2-s) / 2} \max \left(h_{P}, k_{P}\right)^{2}|b|_{2,2 /(2-s)}\left\|u_{y}\right\|_{s, 2, \square_{P}}
$$

and with arguments similar to those already used before

$$
\left|\square_{P}\right|^{1 / 2}\left|\left(\delta_{y}^{(1 / 2)} u_{H}\right)_{P^{r l}}-\delta_{y}^{(1 / 2)} u_{P}\right| \leqslant C \max \left(h_{p}, k_{P}\right)^{s}\left|u_{y}\right|_{s, 2, \square_{P}} .
$$

So, we are left with

$$
\sum_{P \in \Omega_{1 / 2}^{x y} \backslash \Gamma_{1 / 2}^{x y}}\left|\square_{P}\right|\left(b(P) \delta_{y}^{(1 / 2)} u_{P}-\frac{1}{k_{P}} \int_{S_{P}} b u_{y} d y\right) \frac{\delta_{x}^{(1 / 2)} \bar{w}_{P^{+}}+\delta_{x}^{(1 / 2)} \bar{w}_{P^{-}}}{2} .
$$

In the next step, we employ the identity

$$
b(P) \delta_{y}^{(1 / 2)} u_{P}-\frac{1}{k_{P}} \int_{S_{P}} b u_{y} d y=\left(b(P)-\frac{1}{k_{P}} \int_{S_{P}} b d y\right) u_{y}(P)+\frac{1}{k_{P}} \int_{S_{P}}(b(P)-b)\left(u_{y}-u_{y}(P)\right) d y .
$$

Since the first member of the right-hand side of (5.16) vanishes for $b=1, x, y$, we find

$$
\left|\square_{P}\right|^{1 / 2}\left|b(P)-\frac{1}{k_{P}} \int_{S_{P}} b d y\right|\left|u_{y}(P)\right| \leqslant C\left|\square_{P}\right|^{-(2-s) / 2} \max \left(h_{P}, k_{P}\right)^{2}|b|_{2,2 /(2-s)}\left\|u_{y}\right\|_{s, 2, \square_{P}},
$$

which leads to the estimate desired. The second member of the right-hand side of (5.16) can be bounded as in (5.13). This, finally, shows that $F_{1}$ can be bounded appropriately. Moreover, we do not need to assume quasi-uniformity in the case $s=2$.

We now come to $F_{2}$ in (5.12). Due to an estimate similar to (5.13), we can firstly replace

$$
b\left(P^{1}\right) \delta_{y}^{(1 / 2)} u_{P^{r}}+b\left(P^{2}\right) \delta_{y}^{(1 / 2)} u_{P^{l}}-b\left(P^{3}\right) \delta_{y}^{(1 / 2)} u_{P^{l}}-b\left(P^{4}\right) \delta_{y}^{(1 / 2)} u_{P^{r}}
$$

by $2\left(b_{P^{12}}-b_{P^{34}}\right)\left(\delta_{y}^{(1 / 2)} u_{H}\right)_{P r l}$ with the mean values $b_{P^{12}}:=\left(b\left(P^{1}\right)+b\left(P^{2}\right)\right) / 2$ and $b_{P^{34}}:=$ $\left(b\left(P^{3}\right)+b\left(P^{4}\right)\right) / 2$ leading only to an error of the order we need. Reasoning as in (5.14), we secondly replace $b_{P^{12}}$ by $b\left(P^{+}\right)$and $b_{P^{34}}$ by $b\left(P^{-}\right)$. Applying then (5.15) and

$$
\left|\square_{P}\right|^{1 / 2}\left|\delta_{y}^{(1 / 2)} u_{P}-u_{y}(P)\right| \leqslant C \max \left(h_{p}, k_{P}\right)^{s}\left|u_{y}\right|_{s, 2, \square_{P}},
$$


we are left with

$$
F_{2}^{\prime}:=\sum_{P \in \Omega_{1 / 2}^{x y} \backslash \Gamma_{1 / 2}^{x y}} \frac{\left|\square_{P}\right|}{2}\left(\left(b\left(P^{+}\right)-b\left(P^{-}\right)\right) u_{y}(P)-\frac{2}{k_{P}} \int_{S_{P}^{+}} b u_{y} d y+\frac{2}{k_{P}} \int_{S_{P}^{-}} b u_{y} d y\right) \frac{\delta_{x}^{(1 / 2)} \bar{w}_{P^{+}}-\delta_{x}^{(1 / 2)} \bar{w}_{P^{-}}}{2}
$$

to be estimated. With the aid of identity (5.6), the quantity $F_{2}^{\prime}$ can be rewritten as

$$
\begin{aligned}
F_{2}^{\prime}= & \sum_{P \in \Omega_{1 / 2}^{y}} \frac{k_{P}^{2}}{4}\left(-\left(b\left(P^{1}\right)-b\left(P^{4}\right)\right) u_{y}\left(P^{r}\right)+\left(b\left(P^{2}\right)-b\left(P^{3}\right)\right) u_{y}\left(P^{l}\right)-\frac{2}{k_{P}} \sum_{i=1}^{4}(-1)^{i} \int_{S_{P}^{i}} b u_{y} d y\right)+ \\
& +\sum_{P \in \Gamma_{1 / 2}^{y}} \eta_{x}(P)\left(\frac{k_{P}}{2}\left(b\left(P^{I}\right)-b\left(P^{I I}\right)\right) u_{y}(P)-\int_{\Gamma_{P}^{I}} b u_{y} d y+\int_{\Gamma_{P}^{I I}} b u_{y} d y\right) \frac{\bar{w}_{P^{I}}-\bar{w}_{P^{I I}}}{2}+ \\
& +\sum_{P \in \Gamma_{1 / 2}^{x y}} \operatorname{sgn} \eta_{x}(P)\left(\frac{k_{P}}{2}\left(b\left(P^{I}\right)-b\left(P^{I I I}\right)\right) u_{y}(P)-\int_{S_{P}^{I}} b u_{y} d y+\int_{S_{P}^{I I I}} b u_{y} d y\right) \frac{\bar{w}_{P^{I}}-\bar{w}_{P^{I I I}}}{2}=: \\
=: F_{2}^{\prime \prime}+G_{2}+G_{3} . &
\end{aligned}
$$

Here, $G_{2}$ is needed to correct the contributions at the boundary $\Gamma^{y}$ that arise when the sum $F_{2}^{\prime}$, which is taken over all $P \in \Omega_{1 / 2}^{x y}$, is rearranged to a sum over all $P \in \Omega_{1 / 2}^{y}$. Similarly, $G_{3}$ appears in the rearrangement of the sum if $P \in \Omega_{1 / 2}^{y}$ lies on a side of a triangle $\triangle \in \mathcal{T}_{H}^{\text {obl }}$.

With the Cauchy — Schwarz inequality and a relation analogous to (4.9), we find

$$
\left|F_{2}^{\prime \prime}\right| \leqslant C\left(\sum_{P \in \Omega_{1 / 2}^{y}} k_{P}^{3} h_{P}^{-1}\left|F_{P}\right|^{2}\right)^{1 / 2}\left\|P_{H} w_{H}\right\|_{1,2}
$$

where for $P=\left(x_{j}, y_{\ell+1 / 2}\right) \in \Omega_{1 / 2}^{y}$

$$
\begin{gathered}
F_{P}=-\left(b\left(P^{1}\right)-b\left(P^{4}\right)\right) u_{y}\left(P^{r}\right)+\left(b\left(P^{2}\right)-b\left(P^{3}\right)\right) u_{y}\left(P^{l}\right)-\frac{2}{k_{P}} \sum_{i=1}^{4}(-1)^{i} \int_{S_{P}^{i}} b u_{y} d y= \\
=-\int_{y_{\ell}}^{y_{\ell+1}} b_{y}\left(x_{j+1 / 2}, y\right) d y\left(u_{y}\left(P^{r}\right)-u_{y}(P)\right)-\int_{y_{\ell}}^{y_{\ell+1}} b_{y}\left(x_{j-1 / 2}, y\right) d y\left(u_{y}(P)-u_{y}\left(P^{l}\right)\right)- \\
-\left(b\left(P^{1}\right)-b\left(P^{2}\right)+b\left(P^{3}\right)-b\left(P^{4}\right)\right) u_{y}(P)-\frac{2}{k_{P}} \sum_{i=1}^{4}(-1)^{i} \int_{S_{P}^{i}} b u_{y} d y .=: E_{1}+E_{2}+E_{3}+E_{4} .
\end{gathered}
$$

Let $1<s<2$. Since $b \in W^{2,2 /(2-s)}(\Omega) \hookrightarrow \mathcal{C}^{1}(\bar{\Omega})$, we find for $E_{1}$ and $E_{2}$ with the generalised Bramble — Hilbert lemma

$$
\left|E_{1}\right|+\left|E_{2}\right| \leqslant C\left|\square_{P}\right|^{-1 / 2} k_{P} \max \left(h_{P}, k_{P}\right)\left\|b_{y}\right\|_{0, \infty}\left(\left\|u_{y x}\right\|_{s-1,2, \square_{P}}^{2}+\left\|u_{y y}\right\|_{s-1,2, \square_{P}}^{2}\right)^{1 / 2} .
$$

For $E_{3}$, we apply the Bramble - Hilbert lemma with respect to $b \in W^{2,2 /(2-s)}(\Omega)$ as well as the embedding $u_{y} \in H^{s}(\Omega) \hookrightarrow \mathrm{C}(\bar{\Omega})$ and come up with

$$
\left|E_{3}\right| \leqslant C\left|\square_{P}\right|^{-(3-s) / 2} \max \left(h_{P}, k_{P}\right)^{2}|b|_{2,2 /(2-s)}\left\|u_{y}\right\|_{s, 2, \square_{P}} .
$$


Regarding $E_{4}$, we observe that

$$
\begin{aligned}
& E_{4}=-\frac{2}{k_{P}} \sum_{i=1}^{4}(-1)^{i} \int_{S_{P}^{i}}\left(b-b(\bar{P})-b_{x}(\bar{P}) \frac{h_{P}^{i}}{2}-b_{y}(\bar{P})(y-y \bar{P})\right) u_{y} d y-\frac{2}{k_{P}} b(\bar{P}) \sum_{i=1}^{4}(-1)^{i} \int_{S_{P}^{i}} u_{y} d y- \\
& -\frac{2}{k_{P}} b_{y}(\bar{P}) \sum_{i=1}^{4}(-1)^{i} \int_{S_{P}^{i}}\left(y-y_{\bar{P}}\right) u_{y} d y-\frac{2}{k_{P}} b_{x}(\bar{P}) \sum_{i=1}^{4}(-1)^{i} \frac{h_{P}^{i}}{2} \int_{S_{P}^{i}} u_{y} d y=: E_{41}+E_{42}+E_{43}+E_{44},
\end{aligned}
$$

where $\bar{P}$ is the center of $\square_{P}$ and $h_{P}^{1}:=h_{P}^{4}:=h_{P}, h_{P}^{2}:=h_{P}^{3}:=-h_{P}$. Without going into much detail, all the four sums can be bounded appropriately.

Let $s=2$ such that $b \in W^{2, \infty}(\Omega)$ and $u \in H^{3}(\Omega)$. For $P=\left(x_{j}, y_{\ell+1 / 2}\right) \in \Omega_{1 / 2}^{y}$, we find

$$
\begin{aligned}
F_{P} & =-\int_{x_{j-1 / 2}}^{x_{j+1 / 2}}\left(\frac{2}{k_{P}} \int_{y_{\ell}}^{y_{\ell+1 / 2}}\left(b u_{y}\right)_{x} d y-\left(b\left(x, y_{\ell}\right) u_{y}\left(x, y_{\ell+1 / 2}\right)\right)_{x}-\right. \\
& \left.-\frac{2}{k_{P}} \int_{y_{\ell+1 / 2}}^{y_{\ell+1}}\left(b u_{y}\right)_{x} d y+\left(b\left(x, y_{\ell+1}\right) u_{y}\left(x, y_{\ell+1 / 2}\right)\right)_{x}\right) d x
\end{aligned}
$$

Upon noting the embeddings $u_{y} \in H^{2}(\Omega) \hookrightarrow \mathcal{C}(\bar{\Omega})$ and $u_{y x} \in H^{1}(\Omega) \hookrightarrow L^{1}(S)$ for any line segment $S \subset \bar{\Omega}$, the integrand of the outer integral is well-defined for almost all $x$. After carrying out the differentiation with respect to $x$, we wish to replace $b\left(x, y_{\ell}\right)$ as well as $b\left(x, y_{\ell+1}\right)$ by $b\left(x, y_{\ell+1 / 2}\right)$ and $b_{x}\left(x, y_{\ell}\right)$ as well as $b_{x}\left(x, y_{\ell+1}\right)$ by $b_{x}\left(x, y_{\ell+1 / 2}\right)$. This leads to an error of order $\left(h_{P} k_{P}\right)^{1 / 2}$ since in particular

$$
\begin{gathered}
\left|\int_{x_{j-1 / 2}}^{x_{j+1 / 2}}\left(b\left(x, y_{\ell+1 / 2}\right)-b\left(x, y_{\ell}\right)\right) u_{y x}\left(x, y_{\ell+1 / 2}\right) d x\right| \leqslant \\
\leqslant \frac{k_{P}}{2}\left\|b_{y}\right\|_{0, \infty} \int_{x_{j-1 / 2}}^{x_{j+1 / 2}}\left|u_{y x}\left(x, y_{\ell+1 / 2}\right)\right| d x \leqslant C\left(h_{P} k_{P}\right)^{1 / 2}\left\|b_{y}\right\|_{0, \infty}\left\|u_{y x}\right\|_{1,2, \square_{P}} .
\end{gathered}
$$

Similar estimates apply to the other terms appearing in the replacing. We are thus left with $F_{P}^{\prime}:=-\int_{x_{j-1 / 2}}^{x_{j+1 / 2}}\left(\frac{2}{k_{P}} \int_{y_{\ell}}^{y_{\ell+1 / 2}}\left(b u_{y}\right)_{x} d y-\left(b u_{y}\right)_{x}\left(x, y_{\ell+1 / 2}\right)-\frac{2}{k_{P}} \int_{y_{\ell+1 / 2}}^{y_{\ell+1}}\left(b u_{y}\right)_{x} d y+\left(b u_{y}\right)_{x}\left(x, y_{\ell+1 / 2}\right)\right) d x$.

The integrand of the outer integral is the sum of one-dimensional rectangular rules applied to $\left(b u_{y}\right)_{x}$. With the Cauchy-Schwarz inequality, we have

$$
\begin{gathered}
\int_{x_{j-1 / 2}}^{x_{j+1 / 2}}\left(\frac{2}{k_{P}} \int_{y_{\ell}}^{y_{\ell+1 / 2}}\left(b u_{y}\right)_{x} d y-\left(b u_{y}\right)_{x}\left(x, y_{\ell+1 / 2}\right)\right) d x|=| \int_{x_{j-1 / 2}}^{x_{j+1 / 2}} \frac{2}{k_{P}} \int_{y_{\ell}}^{y_{\ell+1 / 2}}\left(y-y_{\ell}\right)\left(b u_{y}\right)_{x y} d y d x \mid \leqslant \\
\leqslant C\left(h_{P} k_{P}\right)^{1 / 2}\left\|\left(b u_{y}\right)_{x y}\right\|_{0,2, \square_{P}} \leqslant C\left(h_{P} k_{P}\right)^{1 / 2}\|b\|_{2, \infty}\left\|u_{y}\right\|_{2,2, \square_{P}},
\end{gathered}
$$


the estimate for the other part in $F_{P}^{\prime}$ being analogous. This leads to $\left|F_{P}\right| \leqslant C\left(h_{P} k_{P}\right)^{1 / 2} \times$ $\|b\|_{2, \infty}\left\|u_{y}\right\|_{2,2, \square_{P}}$ that gives together with (5.18) the estimate desired (without requiring quasiuniformity).

After all, it remains to estimate the contributions from or near the boundary, i.e.,

$$
G:=G_{1}+G_{2}+G_{3}+S_{\Gamma, H}^{x}\left(b u_{y}, w_{H}\right)
$$

where $G_{1}$ is given by $(4.10), G_{2}, G_{3}$ by $(5.17)$, and $S_{\Gamma, H}^{x}\left(b u_{y}, w_{H}\right)$ by $(5.2)$. We find

$$
\begin{gathered}
G=\frac{1}{4} \sum_{P \in \Gamma_{1 / 2}^{y}} \operatorname{sgn} \eta_{x}(P) k_{P}\left(b\left(P^{I}\right)\left(u_{y}(P)-u_{y}\left(P^{I}\right)\right)-b\left(P^{I I}\right)\left(u_{y}(P)-u_{y}\left(P^{I I}\right)\right)\right)\left(\bar{w}_{P^{I}}-\bar{w}_{P^{I I}}\right)+ \\
+\frac{1}{2} \sum_{P \in \Gamma_{1 / 2}^{x y}} \operatorname{sgn} \eta_{x}(P)\left(\left(\frac{k_{P}}{2}\left(b\left(P^{I}\right)-b\left(P^{I I}\right)\right) u_{y}(P)-\int_{S_{P}^{I}} b u_{y} d y+\int_{S_{P}^{I I I}} b u_{y} d y\right)\left(\bar{w}_{P^{I}}-\bar{w}_{P I I I}\right)+\right. \\
\quad+2\left(\frac{k_{P}}{4}\left(b\left(P^{I}\right)+b\left(P^{I I I}\right)\right) \delta_{y}^{(1 / 2)} u_{P^{I V}}-\int_{S_{P}^{I I}} b u_{y} d y\right)\left(\bar{w}_{P^{I I}}-\bar{w}_{P I I I}\right)+ \\
\left.+\left(\frac{k_{P}}{\left|\Gamma_{P}\right|} \int_{\Gamma_{P}^{I}} b u_{y} d \sigma-\frac{k_{P}}{2}\left(b u_{y}\right)\left(P^{I}\right)-\frac{k_{P}}{\left|\Gamma_{P}\right|} \int_{\Gamma_{P}^{I I}} b u_{y} d \sigma+\frac{k_{P}}{2}\left(b u_{y}\right)\left(P^{I I}\right)\right)\left(\bar{w}_{P^{I}}-\bar{w}_{P I I}\right)\right)=: G^{y}+G^{x y} .
\end{gathered}
$$

For $G^{y}$, the generalised Bramble - Hilbert lemma only yields the suboptimal estimate

$$
\left|u_{y}(P)-u_{y}\left(P^{i}\right)\right| \leqslant C\left|\Gamma_{P}\right|^{-1 / 2}\left|\Gamma_{P}\right|^{\min (s-1 / 2,1)}\left\|u_{y}\right\|_{s-1 / 2,2, \Gamma_{P}}, \quad i \in\{I, I I\} .
$$

Here, we used the fact that $u_{y} \in H^{s}(\Omega) \hookrightarrow H^{s-1 / 2}\left(\Gamma_{P}\right)$ is uniformly continuous on $\Omega$ and $\left|u_{y}\right|_{1,2, \Gamma_{P}} \leqslant C\left\|u_{y}\right\|_{s-1 / 2,2, \Gamma_{P}}$ for $3 / 2<s \leqslant 2$. With $b \in W^{2,2 /(2-s)}(\Omega) \hookrightarrow \mathcal{C}(\bar{\Omega})$ and (4.13), it follows

$$
\left|G^{y}\right| \leqslant C \sum_{P \in \Gamma_{1 / 2}^{y}}\left|\Gamma_{P}\right|^{\min (s, 3 / 2)}\left\|u_{y}\right\|_{s-1 / 2,2, \Gamma_{P}}\left|P_{H} w_{H}\right|_{1 / 2,2, \Gamma_{P}},
$$

and the Cauchy - Schwarz inequality proves the asserted bound that holds for $s=2$ without quasi-uniformity.

Regarding $G^{x y}$, we can write

$$
G^{x y}=\frac{1}{2} \sum_{P \in \Gamma_{1 / 2}^{x y}} \operatorname{sgn} \eta_{x}(P)\left(G_{P, 1}+G_{P, 2}\right),
$$

where

$$
\begin{aligned}
& G_{P, 1}:=\left(\frac{k_{P}}{4}\left(b u_{y}\right)\left(P^{I}\right)+\frac{k_{P}}{4}\left(b u_{y}\right)\left(P^{I V}\right)-\int_{S_{P}^{I}} b u_{y} d y-\frac{k_{P}}{4}\left(b u_{y}\right)\left(P^{I V}\right)-\frac{k_{P}}{4}\left(b u_{y}\right)\left(P^{I I I}\right)+\int_{S_{P}^{I I I}} b u_{y} d y\right) \times \\
& \times\left(\bar{w}_{P^{I}}-\bar{w}_{P^{I I I}}\right)+2\left(\frac{k_{P}}{4}\left(b u_{y}\right)(P)+\frac{k_{P}}{4}\left(b u_{y}\right)\left(P^{V}\right)-\int_{S_{P}^{I I}} b u_{y} d y\right)\left(\bar{w}_{P^{I I}}-\bar{w}_{P I I I}\right)+\left(\frac{k_{P}}{\left|\Gamma_{P}\right|} \int_{\Gamma_{P}^{I}} b u_{y} d \sigma-\right. \\
& \left.-\frac{k_{P}}{4}\left(b u_{y}\right)\left(P^{I}\right)-\frac{k_{P}}{4}\left(b u_{y}\right)(P)-\frac{k_{P}}{\left|\Gamma_{P}\right|} \int_{\Gamma_{P}^{I I}} b u_{y} d \sigma+\frac{k_{P}}{4}\left(b u_{y}\right)(P)+\frac{k_{P}}{4}\left(b u_{y}\right)\left(P^{I I}\right)\right)\left(\bar{w}_{P^{I}}-\bar{w}_{P^{I I}}\right),
\end{aligned}
$$




$$
\begin{gathered}
G_{P, 2}:=\frac{k_{P}}{4}\left(2\left(b\left(P^{I}\right)-b\left(P^{I I}\right)\right) u_{y}(P)-\left(b u_{y}\right)\left(P^{I}\right)+\left(b u_{y}\right)\left(P^{I I I}\right)\right)\left(\bar{w}_{P^{I}}-\bar{w}_{P I I I}\right)+ \\
+\frac{k_{P}}{2}\left(\left(b\left(P^{I}\right)+b\left(P^{I I I}\right)\right) \delta_{y}^{(1 / 2)} u_{P^{I V}}-\left(b u_{y}\right)(P)-\left(b u_{y}\right)\left(P^{V}\right)\right)\left(\bar{w}_{P^{I I}}-\bar{w}_{P^{I I I}}\right)+ \\
\left.+\frac{k_{P}}{4}\left(\left(b u_{y}\right)\left(P^{I I}\right)-\left(b u_{y}\right)\left(P^{I}\right)\right)\right)\left(\bar{w}_{P^{I}}-\bar{w}_{P^{I I}}\right) .
\end{gathered}
$$

As in (5.7), $G_{P, 1}$ can be optimally estimated (without quasi-uniformity if $s=2$ ). Since $b \in W^{2,2 /(2-s)}(\Omega) \hookrightarrow \mathrm{C}^{1}(\bar{\Omega})$ and $u_{y} \in H^{s}(\Omega) \hookrightarrow H^{s-1 / 2}\left(\Gamma_{P}\right) \hookrightarrow \mathrm{C}\left(\overline{\Gamma_{P}}\right)$, we have

$$
k_{P}\left|b\left(P^{I}\right)-b\left(P^{I I}\right)\right|\left|u_{y}(P)\right| \leqslant C k_{P}\left|\Gamma_{P}\right|^{1 / 2}|b|_{1, \infty}\left\|u_{y}\right\|_{s-1 / 2,2, \Gamma_{P}} .
$$

In $G_{P, 2}$, reasoning as in the first part of this proof, we can replace $\left(b\left(P^{I}\right)+b\left(P^{I I I}\right)\right) / 2$ by $b\left(P^{I V}\right)$ and afterwards $\delta_{y}^{(1 / 2)} u_{P^{I V}}$ by $u_{y}\left(P^{I V}\right)$ (see also (5.5)) as this leads to an error that can be bounded optimally. If $Q$ and $R \in \bar{\triangle}_{P}$ are two points lying on a straight line in $\bar{\triangle}_{P}$, then the Bramble - Hilbert lemma furnishes (similarly as in (5.9), (5.10))

$$
k_{P}\left|\left(b u_{y}\right)(Q)-\left(b u_{y}\right)(R)\right| \leqslant C\left|\Gamma_{P}\right|\left\|u_{y}\right\|_{1,2, \triangle_{P}} .
$$

This, together with (5.8) and (4.13), shows that

$$
\left|G_{P, 2}\right| \leqslant C\left(\left|\Gamma_{P}\right|\|u\|_{2,2, \triangle_{P}}+\left|\Gamma_{P}\right|^{3 / 2}\left\|u_{y}\right\|_{s-1 / 2,2, \Gamma_{P}}\right)\left(\left|P_{H} w_{H}\right|_{1 / 2,2, \Gamma_{P}}^{2}+\left\|P_{H} w_{H}\right\|_{1,2, \triangle_{P}}^{2}\right)^{1 / 2}
$$

if $\left\{\bar{\Omega}_{H}\right\}_{H \in \Lambda}$ is quasi-uniform for $s \in(1,2)$ (which is not needed for $s=2$ ). The assertion now follows with Lemma 5.2 .

Let us now consider $\tau_{H}^{(f)}$; the terms with first-order derivatives will be studied afterwards.

Lemma 5.5. Let $s \in(1,2], u \in H^{s}(\Omega), f \in W^{2,2 /(2-s)}(\Omega)$, and assume that $\left\{\bar{\Omega}_{H}\right\}_{H \in \Lambda}$ is quasi-uniform if $s \neq 2$. Then for all $w_{H} \in W_{H}$

$$
\begin{gathered}
\left|\tau_{H}^{(f)}\left(w_{H}\right)\right| \leqslant C\left(\sum_{P \in \Omega_{1 / 2}^{x y} \backslash \Gamma_{1 / 2}^{x y}}\left(\operatorname{diam} \square_{P}\right)^{2 s}\|u\|_{s, 2, \square_{P}}^{2}+\right. \\
\left.+\sum_{P \in \Gamma_{1 / 2}^{x y}}\left(\left|\Gamma_{P}\right|^{2 s}\|u\|_{s, 2, \triangle_{P}}^{2}+\left|\Gamma_{P}\right|^{3}\|u\|_{1,2, \triangle_{P}}^{2}\right)\right)^{1 / 2}\left\|P_{H} w_{H}\right\|_{1,2} \leqslant C H_{\max }^{s}\|u\|_{s, 2}\left\|P_{H} w_{H}\right\|_{1,2} .
\end{gathered}
$$

Proof. Starting from (4.4), a straightforward calculation shows that for $w_{H} \in W_{H}$

$$
\tau_{H}^{(f)}\left(w_{H}\right)=\sum_{P \in \Omega_{1 / 2}^{x y} \backslash \Gamma_{1 / 2}^{x y}} \sum_{i=1}^{4}\left|\square_{P}^{(i)}\right|\left((f u)\left(P^{(i)}\right)-\frac{1}{\left|\square_{P}^{(i)}\right|} \int_{\square_{P}^{(i)}} f u d V\right) \bar{w}_{P^{(i)}}+\frac{1}{4} \sum_{P \in \Gamma_{1 / 2}^{x y}}\left|\triangle_{P}\right| F_{P},
$$

where

$$
\begin{aligned}
F_{P}: & =\left((f u)\left(P^{I}\right)-\frac{4}{\left|\triangle_{P}\right|} \int_{\triangle_{P}^{I}} f u d V\right) \bar{w}_{P^{I}}+\left((f u)\left(P^{I I}\right)-\frac{4}{\left|\triangle_{P}\right|} \int_{\triangle_{P}^{I I}} f u d V\right) \bar{w}_{P^{I I}}+ \\
& +2\left((f u)\left(P^{I I I}\right)-\frac{2}{\left|\triangle_{P}\right|} \int_{\square_{P}^{I I I}} f u d V\right) \bar{w}_{P}^{I I I}=: F_{P}^{I} \bar{w}_{P}^{I}+F_{P}^{I I} \bar{w}_{P}^{I I}+F_{P}^{I I I} \bar{w}_{P^{I I I}} .
\end{aligned}
$$


For bounding the sum over all $P \in \Omega_{1 / 2}^{x y} \backslash \Gamma_{1 / 2}^{x y}$, we follow the lines of the proofs of [11, Lemmata 5.6, 5.7] giving the estimate desired. Regarding $F_{P}$, we observe that

$F_{P}=\left(3 F_{P}^{I}+3 F_{P}^{I I}+F_{P}^{I I I}\right) \bar{w}_{P I I I}+3 F_{P}^{I}\left(\bar{w}_{P^{I}}-\bar{w}_{P I I I}\right)+3 F_{P}^{I I}\left(\bar{w}_{P I I}-\bar{w}_{P I I I}\right)-2 F_{P}^{I} \bar{w}_{P^{I}}-2 F_{P}^{I I} \bar{w}_{P I I}$.

Upon noting that $3 F_{P}^{I}+3 F_{P}^{I I}+F_{P}^{I I I}$ vanishes for $f u=1, x, y$, the generalised Bramble Hilbert lemma together with a first-order Taylor expansion of $f$ enables us to verify the estimate

$$
\begin{gathered}
\left|\triangle_{P}\right|^{1 / 2}\left|3 F_{P}^{I}+3 F_{P}^{I I}+F_{P}^{I I I}\right| \leqslant C\left(\left|\Gamma_{P}\right|^{s}\|f\|_{0, \infty}|u|_{s, 2, \triangle_{P}}+\left|\Gamma_{P}\right|^{2}|f|_{1, \infty}|u|_{1,2, \triangle_{P}}+\right. \\
\left.+\left|\triangle_{P}\right|^{-(2-s) / 2}\left|\Gamma_{P}\right|^{2}\|f\|_{2,2 /(2-s)}\|u\|_{1,2, \triangle_{P}}\right) \leqslant C\left|\Gamma_{P}\right|^{s}\|u\|_{s, 2, \triangle_{P}} .
\end{gathered}
$$

This, together with $\left|\triangle_{P}\right|^{1 / 2}\left|w_{P I I I}\right| \leqslant C\left\|P_{H} w_{H}\right\|_{0,2, \triangle_{P}}$, yields the estimate desired that holds without assuming quasi-uniformity if $s=2$.

Since $f \in W^{2,2 /(2-s)}(\Omega) \hookrightarrow \mathcal{C}^{1}(\bar{\Omega})$, we find as usual

$$
\left|F_{P}^{i}\right| \leqslant C\left|\triangle_{P}\right|^{-1 / 2}\left|\Gamma_{P}\right|\|u\|_{1,2, \triangle_{P}}, \quad i \in\{I, I I, I I I\} .
$$

Because of

$$
h_{P}^{1 / 2} k_{P}^{-1 / 2}\left|w_{P I}-w_{P I I I}\right|+k_{P}^{1 / 2} h_{P}^{-1 / 2}\left|w_{P I I}-w_{P I I I}\right| \leqslant C\left|P_{H} w_{H}\right|_{1,2, \triangle_{P}},
$$

it follows (without assuming quasi-uniformity)

$$
\left|\triangle_{P}\right|\left|F_{P}^{i}\right|\left|w_{P^{i}}-w_{P I I I}\right| \leqslant C\left|\Gamma_{P}\right|^{2}\|u\|_{1,2, \triangle_{P}}\left|P_{H} w_{H}\right|_{1,2, \triangle_{P}}, \quad i \in\{I, I I\},
$$

which leads to the optimal estimate. Because of (5.3), we also find

$$
\left|\triangle_{P}\right|\left|F_{P}^{i}\right|\left|w_{P^{i}}\right| \leqslant C\left|\Gamma_{P}\right|^{3 / 2}\|u\|_{1,2, \triangle_{P}}\left\|P_{H} w_{H}\right\|_{0,2, \Gamma_{P}}, \quad i \in\{I, I I\} .
$$

This leads to the local estimate asserted. The global estimate follows with Lemma 5.2.

For the terms with first-order derivatives, we obtain the following result.

Lemma 5.6. Let $s \in(1,2], u \in H^{1+s}(\Omega), d \in W^{2,2 /(2-s)}(\Omega)$, and assume that $\left\{\bar{\Omega}_{H}\right\}_{H \in \Lambda}$ is quasi-uniform if $s \neq 2$. Then for all $w_{H} \in W_{H}$

$$
\begin{aligned}
\left|\tau_{H}^{(d)}\left(w_{H}\right)\right| & \leqslant C\left(\sum_{P \in \Omega_{1 / 2}^{x}}\left(\operatorname{diam} \square_{P}\right)^{2 s}\left\|u_{x}\right\|_{s, 2, \square_{P}}^{2}+\sum_{P \in \Gamma_{1 / 2}^{x y}}\left(\left|\Gamma_{P}\right|^{2 s}\left\|u_{x}\right\|_{s, 2, \triangle_{P}}^{2}+\left|\Gamma_{P}\right|^{3}\left\|u_{x}\right\|_{1,2, \triangle_{P}}^{2}+\right.\right. \\
& \left.\left.+\left|\Gamma_{P}\right|^{3}\left\|u_{x}\right\|_{s-1 / 2,2, \Gamma_{P}}^{2}\right)\right)^{1 / 2}\left\|P_{H} w_{H}\right\|_{1,2} \leqslant C H_{\max }^{\min (s, 3 / 2)}\left\|u_{x}\right\|_{s, 2}\left\|P_{H} w_{H}\right\|_{1,2} .
\end{aligned}
$$

If $\Omega$ is the union of rectangles (i.e., $\Gamma_{1 / 2}^{x y}=\varnothing$ ) the local estimate above yields order $\mathcal{O}\left(H_{\max }^{s}\right)$.

Proof. We have

$$
\tau_{H}^{(d)}\left(w_{H}\right)=d_{H}\left(R_{H} u, w_{H}\right)-\left(R_{H}\left(d u_{x}\right), w_{H}\right)_{H}+\left(R_{H}\left(d u_{x}\right), w_{H}\right)_{H}-\sum_{P \in \bar{\Omega}_{H}} \int_{\square_{P}} d u_{x} d V \bar{w}_{P} .
$$

Since $d \in W^{2,2 /(2-s)}(\Omega)$ and $u_{x} \in H^{s}(\Omega)$, the second difference can be handled exactly as $\tau_{H}^{(f)}$ in Lemma 5.5. For the remaining terms, we find with (4.11) and some calculations

$d_{H}\left(R_{H} u, w_{H}\right)-\left(R_{H}\left(d u_{x}\right), w_{H}\right)_{H}=\sum_{P \in \Omega_{1 / 2}^{x}} h_{P}\left|S_{P}\right|\left(d(P) \delta_{x}^{(1 / 2)} u_{P}\left(P_{H} \bar{w}_{H}\right)(P)-\left(d u_{x} P_{H} \bar{w}_{H}\right)_{P^{r l}}\right)+$ 


$$
+\frac{1}{4} \sum_{P \in \Gamma_{1 / 2}^{x y}}\left|\triangle_{P}\right|\left(\left(d u_{x}\right)\left(P^{I}\right) \bar{w}_{P^{I}}-\left(d u_{x}\right)\left(P^{I I}\right) \bar{w}_{P^{I I}}\right)=: F+G .
$$

With the techniques already known, $F$ can be bounded as asserted. It remains to estimate the contribution $G$ that appears due to the oblique boundary parts. For $P \in \Gamma_{1 / 2}^{x y}$, we have

$$
\begin{gathered}
\left(d u_{x}\right)\left(P^{I}\right) \bar{w}_{P^{I}}-\left(d u_{x}\right)\left(P^{I I}\right) \bar{w}_{P^{I I}}=\left(\left(d u_{x}\right)\left(P^{I}\right)-\left(d u_{x}\right)\left(P^{I I}\right)\right) \bar{w}_{P^{I}}+ \\
+\left(d u_{x}\right)\left(P^{I I}\right)\left(\bar{w}_{P^{I}}-\bar{w}_{P^{I I}}\right)=: G_{P, 1}+G_{P, 2} .
\end{gathered}
$$

Since $d \in W^{2,2 /(2-s)}(\Omega) \hookrightarrow \mathrm{C}^{1}(\bar{\Omega})$ and $u_{x} \in H^{s}(\Omega) \hookrightarrow H^{s-1 / 2}\left(\Gamma_{P}\right)$, we find from the generalised Bramble - Hilbert lemma together with (5.3)

$$
\begin{aligned}
\left|\triangle_{P}\right|\left|G_{P, 1}\right| & \leqslant C\left|\triangle_{P}\right|\left|\Gamma_{P}\right|^{-1+\min (1, s-1 / 2)}\left|d u_{x}\right|_{\min (1, s-1 / 2), 2, \Gamma_{P}}\left\|P_{H} w_{H}\right\|_{0,2, \Gamma_{P}} \leqslant \\
& \leqslant C\left|\Gamma_{P}\right|^{\min (2, s+1 / 2)}\|d\|_{1, \infty}\left\|u_{x}\right\|_{s-1 / 2,2, \Gamma_{P}}\left\|P_{H} w_{H}\right\|_{0,2, \Gamma_{P}} .
\end{aligned}
$$

Here, we made use of the fact that for $1<s<3 / 2$

$$
\left|d u_{x}\right|_{s-1 / 2,2, \Gamma_{P}} \leqslant C\left(\|d\|_{0, \infty}\left|u_{x}\right|_{s-1 / 2,2, \Gamma_{P}}+\left|\Gamma_{P}\right|^{3 / 2-s}|d|_{1, \infty}\|u\|_{0,2, \Gamma_{P}}\right) \leqslant C\|d\|_{1, \infty}\left\|u_{x}\right\|_{s-1 / 2,2, \Gamma_{P}}
$$

and for $3 / 2 \leqslant s \leqslant 2$

$$
\left|d u_{x}\right|_{1,2, \Gamma_{P}} \leqslant C\|d\|_{1, \infty}\left\|u_{x}\right\|_{1,2, \Gamma_{P}} \leqslant C\|d\|_{1, \infty}\left\|u_{x}\right\|_{s-1 / 2,2, \Gamma_{P}}
$$

For $G_{P, 2}$, we can only prove a suboptimal estimate. With $u_{x} \in H^{s}(\Omega) \hookrightarrow H^{s-1 / 2}\left(\Gamma_{P}\right) \hookrightarrow$ $\mathrm{e}\left(\overline{\Gamma_{P}}\right)$ and $(4.13)$, it follows

$$
\begin{aligned}
\left|\triangle_{P}\right|\left|G_{P, 2}\right| \leqslant & C\left|\triangle_{P}\right|\left|\Gamma_{P}\right|^{-1 / 2}\|d\|_{0, \infty}\left\|u_{x}\right\|_{s-1 / 2,2, \Gamma_{P}}\left|P_{H} w_{H}\right|_{1 / 2,2, \Gamma_{P}} \leqslant \\
& \leqslant C\left|\Gamma_{P}\right|^{3 / 2}\left\|u_{x}\right\|_{s-1 / 2,2, \Gamma_{P}}\left|P_{H} w_{H}\right|_{1 / 2,2, \Gamma_{P}} .
\end{aligned}
$$

The foregoing estimates are again valid for $s=2$ without assuming quasi-uniformity. The assertion follows from the application of the Cauchy - Schwarz inequality.

Proof of Theorem 2.2. The assertion of Theorem 2.2 is an immediate consequence of the lemmata in this section together with Proposition 2.1, since for the terms with the coefficients $c$ and $e$ analogous results hold as for $a$ and $d$, respectively. Moreover, we have $\|u\|_{s, 2, \Gamma_{P}} \leqslant C\|u\|_{1 / 2+s, 2, \Gamma_{P}}$ as well as $\sum_{P \in \Gamma_{1 / 2}}\|u\|_{1 / 2+s, 2, \Gamma_{P}}^{2} \leqslant\|u\|_{1+s, 2}^{2}$.

\section{References}

1. R. E. Bank and J.Xu, Asymptotically exact a posteriori error estimators, I. Grids with superconvergence, SIAM J. Numer. Anal., 41 (2003), no. 6, pp. 2294-2312.

2. R.E. Bank and J.Xu, Asymptotically exact a posteriori error estimators, II. General unstructured grids, SIAM J. Numer. Anal., 41 (2003), no. 6, pp. 2313-2332.

3. S. Barbeiro, J. A. Ferreira, and R. D. Grigorieff, Supraconvergence of a finite difference scheme for solutions in $H^{s}(0, L)$, IMA J. Numer. Anal., 25 (2005), no. 4, pp. 797-811.

4. S. Barbeiro, J. A. Ferreira, and J. Brandts, Superconvergence of piecewise linear semi-discretizations for parabolic equations with nonuniform triangulations, J. Math. Fluid Mech., 7 (2005), suppl. 2, pp. S192S214.

5. D. Bojović, Convergence in $W_{2}^{1,1 / 2}$ norm of finite difference method for parabolic problem, CMAM, 3 (2003), no. 1, pp. 45-58. 
6. D. Bojović and B. S. Jovanović, Fractional order convergence rate estimates of finite difference method on nonuniform meshes, CMAM, 1 (2001), no. 3, pp. 213-221.

7. J.H.Brandts, Superconvergence phenomena in finite element methods, Proefschrift, Universiteit Utrecht, 1995 .

8. J.H. Brandts and M. Kř́ížek, Superconvergence of tetrahedral quadratic finite elements, J. Comput. Math., 23 (2005), no 1, pp. 27-36.

9. L. Chen, Superconvergence of tetrahedral linear finite elements, Int. J. Numer. Anal. Model., 3 (2006), no. 3, pp. 273-282.

10. T. Dupont and R. Scott, Polynomial approximation of functions in Sobolev spaces, Math. Comp., 34 (1980), no. 150, pp. 441-463.

11. E. Emmrich and R.D. Grigorieff, Supraconvergence of a finite difference scheme for elliptic third kind boundary value problems in fractional order Sobolev spaces, CMAM, 6 (2006), no. 2, pp. 154-177.

12. J. A. Ferreira and R. D. Grigorieff, On the supraconvergence of elliptic finite difference schemes, Appl. Numer. Math., 28 (1998), no. 2-4, pp. 275-292.

13. J. A. Ferreira and R. D. Grigorieff, Supraconvergence and supercloseness of a scheme for elliptic equations on non-uniform grids, Numer. Funct. Anal. Optimiz., 27 (2006), no. 5-6, pp. 539-564.

14. B. García-Archilla, A supraconvergent scheme for the Korteweg-de Vries equation, Numer. Math., 61 (1992), no. 3, pp. 292-310.

15. W. Hackbusch, Theory and numerical treatment of elliptic differential equations, Springer, Berlin, 1992.

16. I. Hlaváček and M. Křížek, On a superconvergent finite element scheme for elliptic systems, I. Dirichlet boundary condition, Apl. Mat., 32 (1987), no. 2, pp. 131-154.

17. I. Hlaváček and M. Kř́̌žek, On a superconvergent finite element scheme for elliptic systems, II. Boundary conditions of Newton's or Neumann's type, Apl. Mat., 32 (1987), no. 3, pp. 200-213.

18. I. Hlaváček and M. Křížek, On a superconvergent finite element scheme for elliptic systems, III. Optimal interior estimates, Apl. Mat., 32 (1987), no. 4, pp. 276-289.

19. I. Hlaváček, M. Kř́̌́žek, and V. Pištora, How to recover the gradient of linear elements on nonuniform triangulations, Apl. Mat., 41 (1996), no. 4, pp. 241-267.

20. V.P.Il'in, The properties of some classes of differentiable functions of several variables defined in an $n$-dimensional region, Proc. Steklov Inst. Math., 81 (1969), pp. 91-256 (Trudy Mat. Inst. Steklov, 66 (1962), pp. 227-363).

21. B. S. Jovanović, Finite difference schemes for partial differential equations with weak solutions and irregular coefficients, CMAM, 4 (2004), no. 1, pp. 48-65.

22. B. S. Jovanović, The finite difference method for boundary-value problems with weak solutions, Posebna Izdanja, 16. Matematički Institut u Beogradu, Belgrade, 1993.

23. B. S. Jovanović, L. D. Ivanović, and E. E. Süli, Convergence of a finite-difference scheme for secondorder hyperbolic equations with variable coefficients, IMA J. Numer. Anal., 7 (1987), no. 1, pp. 39-45.

24. B. S. Jovanović and P. P. Matus, Estimation of the convergence rate of difference schemes for elliptic problems, Comput. Math. Math. Phys., 39 (1999), pp.56-64 (Zh. Vychisl. Mat. Mat. Fiz., 39 (1999), no. 1, pp. 61-69).

25. B. S. Jovanović, L. D. Ivanović, and E. E.Süli, Convergence of finite-difference schemes for elliptic equations with variable coefficients, IMA J. Numer. Anal., 7 (1987), pp. 301-305.

26. B.S. Jovanović and B.Z. Popović, Some convergence rate estimates for finite difference schemes, Mat. Vesnik, 49 (1997), pp. 249-256.

27. B. S. Jovanović and B.Z. Popović, Convergence of a finite difference scheme for the third boundaryvalue problem for an elliptic equation with variable coefficients, CMAM, 1 (2001), no. 4, pp. 356-366.

28. M. Kř́̌žek and P. Neittaanmäki, On superconvergence techniques, Acta Applic. Math., 9 (1987), pp. 175-198.

29. M. Kř́̌žek and P. Neittaanmäki, Superconvergence phenomenon in the finite element method arising from averaging gradients, Numer. Math., 45 (1984), pp. 105-116.

30. M. Křížek and P. Neittaanmäki, On a global superconvergence of the gradient of linear triangular elements, J. Comput. Appl. Math., 18 (1987), pp. 221-233.

31. M. Křížek and P. Neittaanmäki, Bibliography on superconvergence, in: Finite element methods (M. Křížek et al., eds.), M. Dekker, New York, 1998, pp. 315-348.

32. A. Kufner, O. John, and S. Fučík, Function Spaces, Noordhoff Intern. Publ., Leyden, 1977.

33. L. R. Lalović, Approximation of generalized solutions of the third boundary value problem for elliptic equation with variable coefficients. Zb. radova (Kragujevcu), 19 (1997), pp. 53-65. 
34. R. D. Lazarov, V.L. Makarov, and W. Weinelt, On the convergence of difference schemes for the approximation of solutions $u \in W_{2}^{m}(m>0.5)$ of elliptic equations with mixed derivatives, Numer. Math., 44 (1984), no. 2, pp. 223-232.

35. N. Levine, Superconvergent recovery of the gradient from piecewise linear finite-element approximations, IMA J. Numer. Anal., 5 (1985), pp. 407-427.

36. Q. Lin, Superclose FE-theory becomes a table of integrals, in: Finite element methods (M. Křížek et al., eds.), M. Dekker, New York, 1998, pp. 217-225.

37. Q. Lin, L. Tobiska, and A.Zhou, Superconvergence and extrapolation of non-conforming finite elements applied to the Poisson equation, IMA J. Numer. Anal., 25 (2005), no 1, pp. 160-181.

38. L. Liu, T. Liu, M. Křížek, T. Lin, and S. Zhang, Global superconvergence and a posteriori error estimators of the finite element method for a quasi-linear elliptic boundary value problem of nonmonotone type, SIAM J. Numer. Anal., 42 (2004), no. 4, pp. 1729-1744.

39. P. Matus, Stability of difference schemes for nonlinear time-dependent problems, CMAM, 3 (2003), no. 2, pp. 313-329.

40. P. Matus and G. Martsynkevich, Monotone and economical difference schemes on nonuniform grids for a multidimensional parabolic equation with a boundary condition of the third kind, CMAM 4 (2004), no 3, pp. 350-367.

41. P. Matus and I. Rybak, Difference schemes for elliptic equations with mixed derivatives, CMAM, 4 (2004), no. 4, pp. 494-505. 2000 .

42. W. McLean, Strongly elliptic systems and boundary integral equations, University Press, Cambridge,

43. K.W. Morton and E. Süli, Evolution-Galerkin methods and their supraconvergence, Numer. Math. 71 (1995), no. 3, pp. 331-355.

44. L. A. Oganesyan and L.A. Rukhovets, Study of the rate of convergence of variational difference schemes for second-order elliptic equations in a two-dimensional field with a smooth boundary, USSR Comput. Math. Math. Phys., 9 (1972), pp. 153-183 (Zh. Vychisl. Mat. Mat. Fiz., 9 (1969), no. 5, pp. 11021120).

45. F. Pascal, On supra-convergence of the finite volume method, ESAIM: Proceedings, submitted.

46. B. Popović, Convergence of finite-difference schemes for Poisson's equation with boundary condition of the third kind, Mat. Vesnik, 47 (1995), pp. 23-30.

47. U. Risch, Superconvergence of a nonconforming low order finite element, Appl. Numer. Math., 54 (2005), pp. 324-338.

48. V. Thomée, J.Xu and N.Y.Zhang, Superconvergence of the gradient in piecewise linear finiteelement approximation to a parabolic problem, SIAM J. Numer. Anal., 26 (1989), no. 3, pp. 553-573.

49. P. N. Vabishchevich, A. A. Samarskij, and P.P. Matus, Second-order accurate finite-difference schemes on nonuniform grids, Comput. Math. Math. Phys., 38 (1998), pp. 399-410 (Zh. Vychisl. Mat. Mat. Fiz., 38 (1998), no. 3, pp. 413-424).

50. L. B. Wahlbin, Superconvergence in Galerkin finite element methods, Lect. Notes in Math. 1605, Springer, Berlin, 1995.

51. B. Wendroff and A. B. White Jr., Some supraconvergent schemes for hyperbolic equations on irregular grids, in: Nonlinear hyperbolic equations - theory, computation methods, and applications, Aachen 1988 (J. Ballmann and R. Jeltsch, eds.), Notes Numer. Fluid Mech. 24, Vieweg, Braunschweig, 1989, pp. 671-677.

52. J.R. Whiteman and G. Goodsell, Some gradient superconvergence results in the finite element method, in: Numerical analysis and parallel processing (P. R. Turner, ed.), Lect. Notes in Math. 1397, Springer, Berlin, 1987, pp. 182-260.

53. Q.Zhu, A survey of superconvergence techniques in finite element methods, in: Finite element methods (M. Křížek et al., eds.), M. Dekker, New York, 1998, pp. 287-302.

54. A. Zlotnik, On superonvergence of a gradient for finite element methods for an elliptic equation with the nonsmooth right-hand side, CMAM, 2 (2002), no. 3, pp. 295-321. 